\title{
Influences of cloud heterogeneity on cirrus optical properties retrieved from the visible and near-infrared channels of MODIS/SEVIRI for flat and optically thick cirrus clouds
}

\author{
Yongbo Zhou, Xuejin Sun*, Riwei Zhang, Chuanliang Zhang, Haoran Li, Junhao Zhou, \\ Shaohui Li
}

College of Meteorology and Oceanography, PLA University of Science and Technology, Nanjing, China

\begin{abstract}
The influences of three-dimensional radiative effects and horizontal heterogeneity effects on the retrieval of cloud optical thickness (COT) and effective diameter (De) for cirrus clouds are explored by the SHDOM radiative transfer model. The stochastic cirrus clouds are generated by the Cloudgen model based on the Atmospheric Radiation Measurement program data. Incorporating a new ice cloud spectral model, we evaluate the retrieval errors for two solar zenith angles (SZAs) $\left(30^{\circ}\right.$ and $\left.60^{\circ}\right)$, four solar azimuth angles $\left(0^{\circ}\right.$, $45^{\circ}, 90^{\circ}$, and $180^{\circ}$ ), and two sensor settings (Moderate Resolution Imaging Spectrometer (MODIS) onboard Aqua and Spinning Enhanced Visible and Infrared Imager (SEVIRI) onboard METEOSAT-8). The domain-averaged relative error of $\mathrm{COT}(\mu)$ ranges from $-24.1 \%$ to $-1.0 \%\left(\mathrm{SZA}=30^{\circ}\right)$ and from $-11.6 \%$ to $3.3 \%\left(\mathrm{SZA}=60^{\circ}\right)$, with the uncertainty within $7.5 \% \sim 12.5 \%\left(\mathrm{SZA}=30^{\circ}\right)$ and $20.0 \% \sim 27.5 \%\left(\mathrm{SZA}=60^{\circ}\right)$. For the SZA of $60^{\circ}$ only, the relative error and uncertainty are parameterized by the retrieved COT by linear functions, providing bases to correct the retrieved COT and estimate their uncertainties. Besides, De is overestimated by $0.7 \mu \mathrm{m} \sim 15.0 \mu \mathrm{m}$ on the domain average, with the corresponding uncertainty within $6.7 \mu \mathrm{m}$ $\sim 26.5 \mu \mathrm{m}$. The retrieval errors show no discernible dependence on solar azimuth angle due to the flat tops and full coverage of the cirrus samples. The results are valid only for the two samples and for the specific spatial resolution of the radiative transfer simulations.
\end{abstract}

Keywords: three-dimensional radiative effects; horizontal heterogeneity effects; stochastic cirrus clouds; cloud optical thickness; MODIS; SEVIRI

\section{Introduction}

Cirrus clouds are ubiquitous in the atmosphere with their globally averaged occurrence frequency ranging from $16 \%$ to $27 \%$ (Stubenrauch et al. 2006; Sassen et al. 2008) and reaching up to $70 \%$ as a maximum in the tropics (Nazaryan et al. 2008). Existing research indicates that cirrus clouds are an important

\footnotetext{
${ }^{1}$ Nonstandard abbreviations: Cloud Optical Thickness (COT), Effective Diameter (De), Independent Pixel Approximation (IPA), Ice Water Content (IWC), Near-infrared (NIR), Plane Parallel Approximation (PPA), Severely Roughened Aggregated Columns (SRAC), Thermal infrared (TIR), Three-dimensional (3-D), Visible (VIS).

* Corresponding author.

E-mail address: xuejin.sun@outlook.com (X. Sun).
} 
modulator of the radiation and energy balance of the earth-atmosphere system (Cox, 1971; Liou, 1986), and that the modulation effects are closely related to the cloud optical properties (Jensen et al. 1994; Mcfarquhar and Heymsfield, 1998. Dupont and Haeffelin, 2008).

The cloud optical properties, including cloud optical thickness (COT) and effective diameter (De), are frequently retrieved by the spaceborne passive sensors (Marshak et al., 2006; Pandey et al. 2012; Wang et al. 2016). The retrieval algorithms of the passive sensors make two basic assumptions about the realistic heterogeneous cloud fields, the plane parallel approximation (PPA) and independent pixel approximation (IPA) (Davis and Marshak, 2010). Because COT and De belong to the same retrieval space, the two assumptions cause errors to the remote sensing of COT and De by the similar mechanisms. The retrieval errors due to PPA and IPA have their roots in the horizontal heterogeneity effects and three-dimensional (3-D) radiative effects (Marshak et al., 2006; Zinner and Mayer, 2006; Kalesse, 2009; Fauchez et al. 2015). First, the scale of the satellite's pixel is usually larger than the cloud subscale in existing research (Zinner and Mayer, 2006; Fauchez et al. 2014; Fauchez et al. 2015). The subscale denotes the scale of the variability in the radiation fields (Marshak et al. 1998). For the visible (VIS) and near-infrared (NIR) bands, the subscale variability of the cloud properties fails the PPA. This introduces errors when inferring the cloud optical properties from the averaged reflectance of the subpixels (measured as the subscale) within a satellite's pixel due to the nonlinear curvature properties of the reflectance-COT function (Marshak et al. 1998; Zinner and Mayer, 2006) and reflectance-De function (Marshak et al., 2006). Second, the interactions between neighboring pixels are neglected when the IPA is adopted. Radiation interactions between adjacent pixels damage the exactly corresponding relationship between satellite measurements and COT/De (Várnai and Marshak, 2001; Kalesse, 2009). On the domain average, the reflectance tends to increase for optically thinner regions, while it decreases for optically thicker regions for small solar zenith angles. This is termed the channeling effect (Davis and Marshak, 2001; Iwabuchi and Hayasaka, 2002; Várnai and Marshak, 2003). The channeling effect contributes to the smoothing of the radiation fields. For larger solar zenith angles, the side illumination effect tends to increase the reflectance for the optically thicker regions, sharpening the radiation fields (Várnai and Marshak, 2003; Zinner and Mayer, 2006). For cloud fields with bumpy tops, shadows and bright slopes further strengthen the sharpening of the radiation fields (Várnai, 1998; Iwabuchi and Hayasaka, 2002). The interactions between the adjacent pixels exhibit asymmetric properties (Faure et al. 2001; Várnai and Marshak, 2003), implying the dependence of the 3-D radiative effects on the sun-view geometries (Várnai and Marshak, 2001; Zinner and Marshak, 2006). For small pixel size, the errors in the retrieval process are mainly attributed to the IPA. However, the errors due to the PPA become dominant for relatively large pixel size (Várnai and Marshak, 2001). The blending effects of the 3-D radiative effects and horizontal heterogeneity effects reveal more uncertainties and errors in the remote sensing procedure. For the thermal infrared (TIR) bands, the 3-D radiative effects still exist in the stochastic cloud fields (Kablick et al. 2011), and the errors due 
to the IPA are inevitable in the remote sensing of cloud optical properties from the TIR bands. A recent study by Fauchez (2015) explored the errors due to the PPA on the remote sensing of cirrus optical properties for the Imaging Infrared Radiometer (IIR) on board CALIPSO (Garnier et al. 2012; Garnier et al 2013) and concluded an underestimation of the COT and an overestimation of De. Such a result could be extrapolated to IIR-like sensors. The influences of the 3-D radiative effects and horizontal heterogeneity effects on the remote sensing of cloud optical properties are distinctly different for different wavelength ranges, which is a problem still being studied (Fauchez et al. 2015). It is noted that the vertical heterogeneity is also an important cause of errors in the retrieval process. Giuseppe (2005) suggested an overestimation of the COT from solar fluxes $(0.3-3.0 \mu \mathrm{m})$ when the vertical heterogeneity of the liquid water content is neglected. Zhang and Platnick (2012) identified the vertical heterogeneity of the cloud field as one of the causes of differences between the De derived from the $3.7 \mu \mathrm{m}$ and $2.1 \mu \mathrm{m}$ bands of MODIS. Apart from these, Fauchez et al. (2015) demonstrated that the vertical heterogeneities help to smooth the horizontal heterogeneity effects (for the TIR bands only).

Some of the research cited above focuses on the liquid water cloud, but the generalized results are thought to be of interest in the research for cirrus clouds. Kalesse (2009) is among the first to quantify the influences of the 3-D radiative effects on the remote sensing of the COT and the effective radius (Re) for cirrus clouds and indicates that the COT is underestimated while Re is overestimated on average. Although the general findings are meaningful, the ice cloud spectral models differ from that adopted in this study. In addition, the errors due to the PPA are not explored in his research.

In this study, the Moderate Resolution Imaging Spectrometer (MODIS) onboard Aqua (Aqua/MODIS, hereafter, MODIS) (Yamamoto et al. 2011) and the Spinning Enhanced Visible and Infrared Imager (SEVIRI) onboard METEOSAT-8 (METEOSAT-8/SEVIRI, hereafter, SEVIRI) (Roebeling et al. 2006) are the focus. The current algorithms of the two sensors do not account for the 3-D radiative effects and horizontal heterogeneity effects on the remote sensing of COT and De, leaving biases and uncertainties in the retrieved cloud optical properties. To address this, we perform the remote sensing process for a series of generalized conditions, and attempt to provide a guideline to correct the retrieved COT and estimate its corresponding uncertainty. Because the vertical heterogeneity is incorporated into the stochastic cloud fields, its effects on the remote sensing process are not analyzed explicitly. The remainder of this study is organized as follows. Section 2 presents the models and data for the construction of the 3-D stochastic cirrus clouds. Section 3 introduces the methodology. Section 4 elaborates on the results and the corresponding analyses. Section 5 summarizes the general conclusions.

\section{Construction of the 3-D stochastic cirrus fields}

The 3-D stochastic cirrus clouds are generated by the cirrus generator termed Cloudgen. The input 
parameters of Cloudgen are derived from the Atmospheric Radiation Measurement (ARM) program data. The single scattering properties of cirrus clouds are derived from the Severely Roughened Aggregated Columns (SRAC) ice cloud spectral model. The details of the relevant models are provided below, along with the data and their processing methods.

\subsection{Cloudgen model}

Cloudgen is designed to simulate 3-D stochastic cirrus fields from two-dimensional observations (in time-height space) of the Ice Water Content (IWC). It excels at simulating the fallstreak geometries and shear-induced mixing characteristics specific to cirrus clouds (Hogan and Kew, 2005). The 3-D distribution of IWC simulated by Cloudgen was validated by radar observations and the results showed good consensus with each other (Kew, 2003; Hogan and Kew, 2005). Therefore, the model was widely used in areas relevant to the 3-D radiative transferring simulations for stochastic cirrus fields (Davis et al. 2007; Zhong et al. 2008).

The input parameters of Cloudgen include the mean and standard deviation of the logarithm of IWC $(\ln I W C)$, the slope and outer scale of the power spectra of $\ln I W C$, the cloud base and top height, the generating level, the falling speed of ice crystals and the wind profile. All these parameters are derived from the ARM program data.

\subsection{ARM program data and their processing methods}

The ARM program data at Manus, Papua, New Guinea (2³'39.64" S, $147^{\circ} 25^{\prime} 31.43^{\prime \prime}$ E) are used to generate the input parameters of Cloudgen. The input parameters can be categorized into three types, 1) the microphysical properties (the statistical characteristics of $\ln I W C$ and parameters derived from it), 2) the macrophysical properties (the cloud base and top height and the generating level), and 3) the atmosphere state (wind profile).

\subsubsection{Microphysical properties}

The IWC data are provided by the ARM continuous microphysical retrieval Value-Added Product, where IWC is converted from the reflectivity $(Z$ ) of a $35-\mathrm{GHz}$ Millimeter Wave Cloud Radar by Equation (1) (Dunn et al. 2011)

$$
I W C=0.097 Z^{0.59}
$$

Similar to the results by Hogan and Illingworth (2003), the probability density function (PDF) of IWC can be fitted to the lognormal distribution expressed by Equation (2)

$$
\frac{d N}{d \ln I W C}=\frac{N_{0}}{\sqrt{2 \pi} \sigma_{\ln I W C}} \exp \left[-\frac{(\ln I W C-\overline{\ln I W C})^{2}}{2 \sigma_{\ln I W C}^{2}}\right]
$$

where $\overline{\ln I W C}$ and $\sigma_{\ln I W C}$ are the mean and standard deviation of $\ln I W C$. The parameter $N$ denotes the number of pixels with the logarithm of IWC ranging from $\ln I W C$ to $\ln I W C+d \ln I W C$. The term $N_{o}$ is the 
integral of $N$ through the whole $\ln I W C$ range. The per-layer PDF of the IWC is fitted to Equation (2), and the corresponding regression coefficient represents the effectiveness of the regression. To guarantee the representativeness of the statistical results, only the layers with regression coefficients larger than 0.7 are thought valid, and the gaps between the valid layers are filled by interpolation. The per-layer $\overline{\ln I W C}$ and $\sigma_{\text {lnIWC }}$ are used to constrain the generation process of the cirrus clouds to produce the lognormal distributions of the IWC at each height.

The falling speed of ice crystals is characterized by $\overline{I W C(z)}$ (per-layer averaged IWC) by Equation (3) (Szczap et al. 2014),

$$
w(z)=0.25 \log _{10}[\overline{I W C(z)}]+1.5
$$

The power spectra of the per-layer $\ln I W C$, which are calculated by regular Fourier spectral analysis in the range space, can be fitted to the power function by Equation (4)

$$
E(k)=E_{0} k^{-\mu}
$$

where $\mu$ is the slope of the power spectra, $E_{0}$ denotes the power for unit wavenumber and $k$ denotes the arbitrary wavenumber, which is the reciprocal of the distance. The IWC data are gridded in time space, and are converted to range space by the wind profile (Kew, 2003; Hogan and Kew, 2005).

The power spectra of $\ln I W C$ are separated into two distinct regions by a threshold value of the wavenumber, and the outer scale is calculated as the reciprocal of the threshold value. A scale break, centering at the outer scale, of the power spectra is exhibited. Notably, the power spectra become rather flat when the scale is larger than the outer scale, while it presents as an approximately constant $\mu$ of around $-5 / 3$ when the scales are smaller than the outer scale (Davis et al. 1996; Hogan and Kew, 2005). Previous research (Davis et al. 1996) demonstrates that the outer scale varies from $20 \mathrm{~km}$ to $60 \mathrm{~km}$ for stratocumulus clouds, which is similar to the results by Kew (2003) and Hogan and Kew (2005) for cirrus clouds. However, Wang and Sassen (2008) also suggest an outer scale ranging from $4 \mathrm{~km}-6 \mathrm{~km}$ for their specific cirrus case. The difference of the outer scale for different cirrus cases is mainly caused by different dynamic processes.

\subsubsection{Macrophysical properties}

The cloud top height is determined as the maximum height when the per-layer ratio of valid pixels to the total pixels is larger than 0.8. A valid pixel denotes a pixel with an IWC larger than $0.001 \mathrm{~g} / \mathrm{m}^{3}$. The cloud base height is determined by a similar method.

The generating level is the reference height to perform the conversion from time to range. Strictly, the generating level can be inferred from the temperature profile by searching for the altitude corresponding to the 
catastrophe point where the temperature gradient turns from a dry adiabatic lapse rate to a smaller value (Kew, 2003). In cases where the temperature profile is not available or is inaccurate, the generating level can be estimated by two other methods, 1) The generating level can be estimated by investigating the trajectories of cirrus fallstreak structures. Because the generating level denotes the altitude where cirrus formation begins, no horizontal displacement should be expected at this altitude. 2) The generating level could be estimated as the cloud top or higher altitude for geometrically thin cirrus clouds (Kew, 2003). For simplicity, the two methods are termed the physical method and empirical method.

\subsubsection{Wind profile}

The time-height cross section of the IWC is converted from time-height space to range-height space by method introduced by Hogan and Kew (2005), which uses the wind profile as an essential input parameter. The wind profile is provided by the ARM interpolated sounding Value-Added Product constructed from measurements of radiosonde, microwave radiometers, and surface meteorological instruments (Troyan, 2013). During the conversion process, the wind profile was mapped onto the IWC data levels by interpolation.

\subsection{The Severely Roughened Aggregated Columns model}

The single scattering optical properties of ice crystals are derived from the Severely Roughened Aggregated Columns (SRAC) ice cloud spectral model, which involves randomly oriented aggregates of solid columns with the size distribution as a Gamma function (Baum et al. 2014). The SRAC model is constructed from field campaigns and data sets developed by Yang et al. (2013). Validation studies indicate that the SRAC model guarantees good consistency between the reflectance simulated by radiative transfer model and CALIPSO measurements (Baum et al. 2014). The single scattering properties of the SRAC model are similar to those of the updated ice cloud model implemented in the MODIS C006 algorithm (Platnick et al. 2015). Therefore, the SRAC ice cloud spectral model is used in relevant research (Yang et al. 2015) and in this study.

It is noteworthy that the scattering phase functions of the SRAC ice cloud spectral model are converted to Wigner-d function expansion coefficients for radiative simulation. Because the phase functions of SRAC model are close to being pathological delta functions at small scattering angles, the Wigner-d functions at high order tend to be oscillating. Accordingly, the errors in the conversion process are amplified, especially for the highly peaked phase functions with magnitudes varying by more than five orders. Therefore, we introduce a weighted least squares method to perform such a conversion. More details about this method are provided in the Appendix. 


\subsection{Two cirrus samples}

Two cirrus samples are selected. Sample 1 was from January 21, 2010, and sample 2 was from February 25, 2010. Fig. 1 shows the time-height cross section of the IWC of the two samples derived from the ARM continuous microphysical retrieval Value-Added Product.

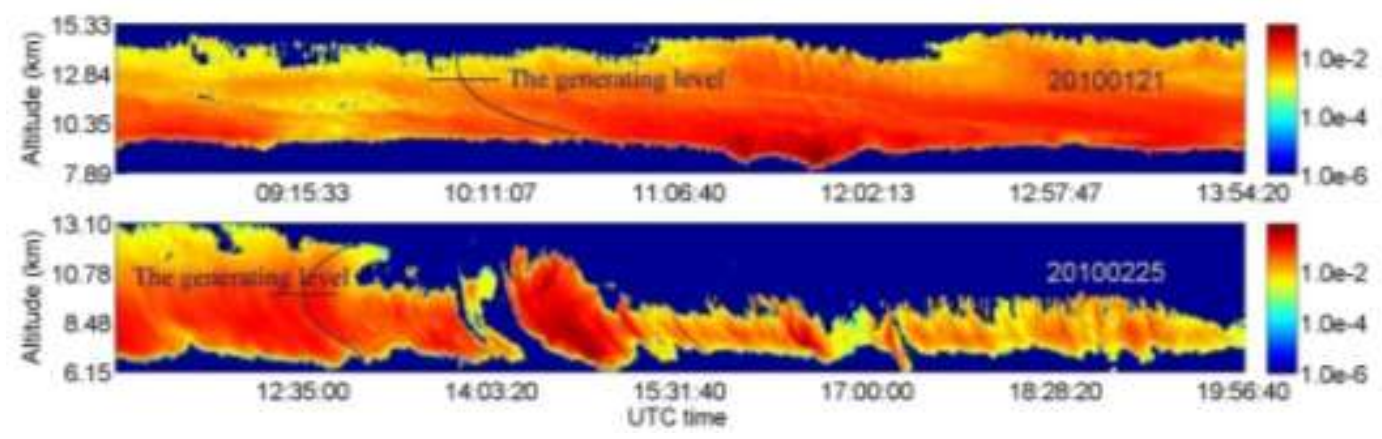

Fig. 1 The time-height cross section of IWC $\left(\right.$ unit: $\left.\mathrm{g} / \mathrm{m}^{3}\right)$. The black curves denote the typical fallstreak structures, and the short horizontal lines denote the generating levels.

To guarantee that the time-converted distance is larger than $60 \mathrm{~km}$ to locate better the outer scale, the time dimension of the two samples span several hours. Consequently, the averaged temperature profile during this period deviates from the true state. Therefore, the generating level has to be determined by the two alternative methods introduced in Section 2.2.2. The trajectories of the fallstreak (black curves in Fig. 1) are similar to the conceptual model of the cirrus uncinus promoted by Heymsfield (1975). By the physical method, the generating level is estimated to be around $12.5 \mathrm{~km}$ for Sample 1 . Sample 2 is geometrically thin. By the empirical method, the generating level is estimated as the cloud top $(8.9 \mathrm{~km})$. More details about the macrophysical properties of the two samples are summarized in Table 1.

Table 1 The macrophysical properties of the two cirrus samples $(\mathrm{km})$

\begin{tabular}{lllll}
\hline Cirrus Sample & Cloud base & Cloud top & Generating level & Outer scale \\
\hline Sample 1 & 9.5 & 13.55 & 12.5 & 20.0 \\
Sample 2 & 7.4 & 8.9 & 8.9 & 25.0 \\
\hline
\end{tabular}

To calculate $\mu$, the power spectra of per-layer $\ln I W C$ are substituted into Equation (4). Due to statistical noise in the energy spectra, it is possible that the regression coefficients are smaller than 0.7 . In such cases, $\mu$ is deemed invalid and is screened out, leaving gaps in particular layers. These gaps are relaxed to the values of the nearest layers. Fig. 2 summarizes the vertical distribution of $\mu$ for the two cirrus samples. Fig. 2 also gives the vertical distribution of $\overline{\ln I W C}, \sigma_{\ln I W C}$, and wind speed. 

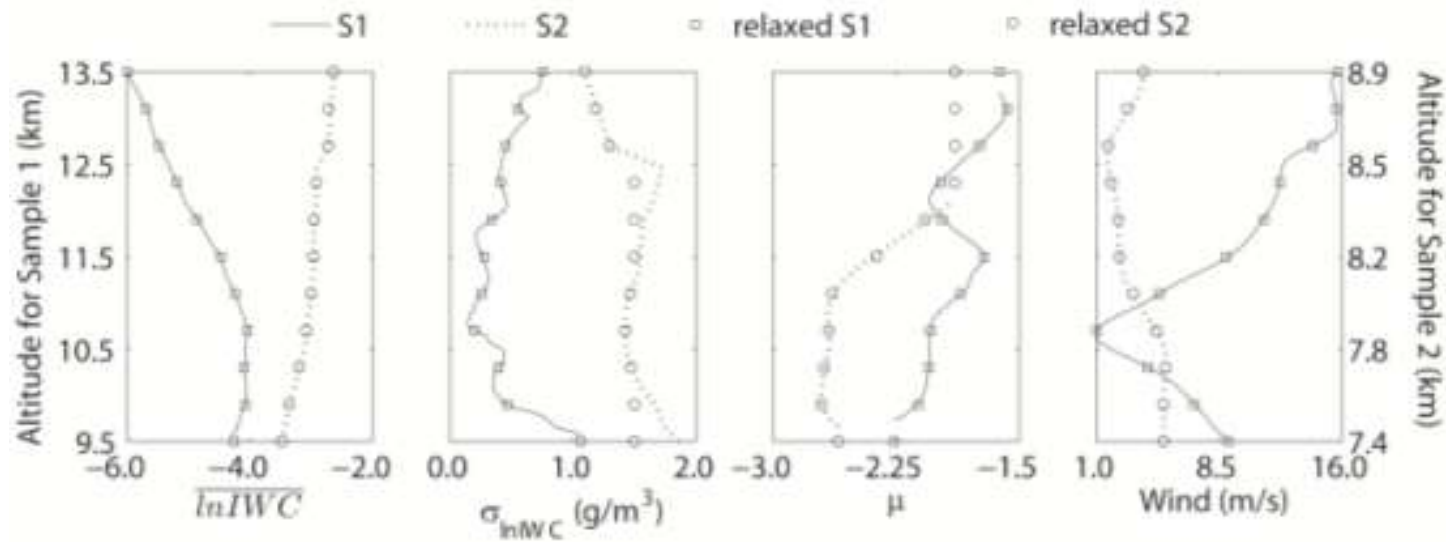

Fig. 2 The microphysical properties of the two cirrus samples. S1 and S2 denote Sample 1 and Sample 2.

The slopes of the power spectra shown in Fig. 2, together with the outer scales summarized in Table 1, are also shown by Fig. 3.
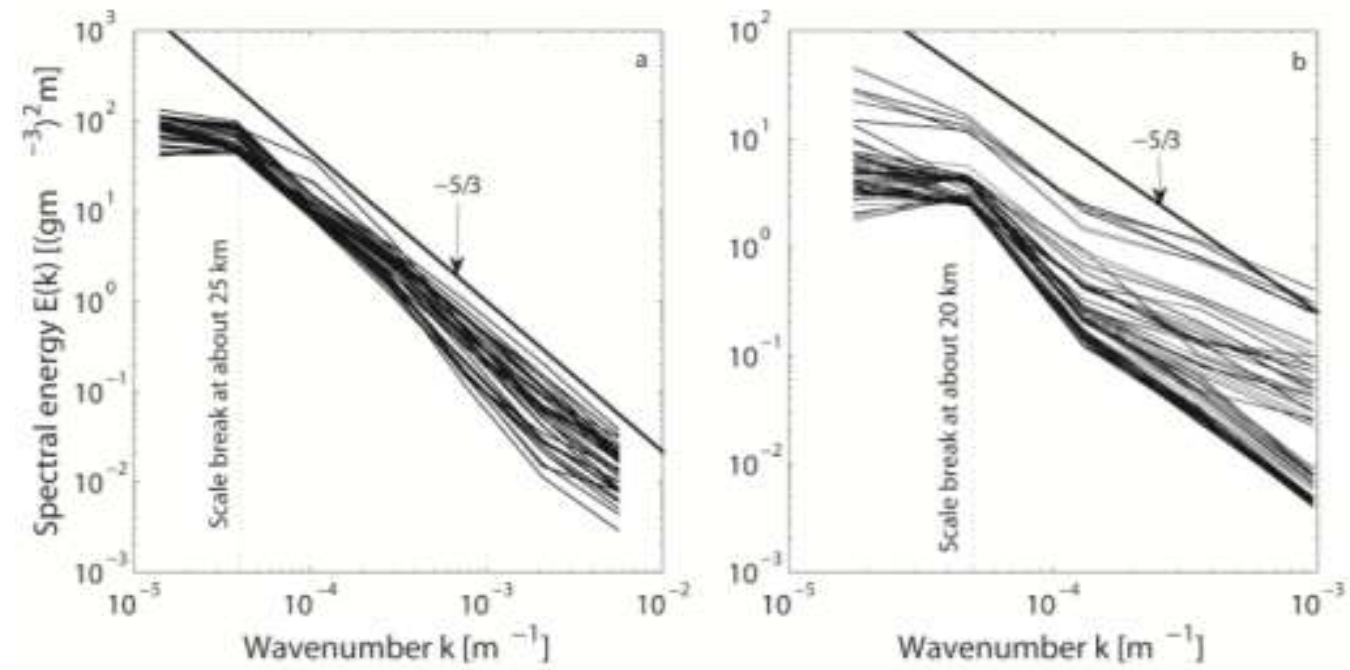

Fig. 3 The power spectra of $\ln I W C$ for the two cirrus samples. a and b denote Sample 1 and Sample 2 separately. The raw power spectra have been averaged such that there are around four points per decade of wavenumber.

For Sample 1, the spectral slope approximates to $-5 / 3$ near the generating level, possibly caused by the turbulence due to convective overturning near the generating level (Hogan and Kew, 2005). Below the generating level, horizontal displacement due to wind shear will lead to a horizontal homogenization of the IWC structure, which reduces the energy spectra at small scales (Hogan and Illingworth, 2003). Therefore, the slope degrades with the decrease of height below the generating level. It is emphasized that the power spectra also vary considerably as a function of gravity wave activity, mesoscale turbulence (Gultepe and Starr, 1995), gravitational sedimentation (Hogan and Kew, 2005), latent heat release due to sublimation (Hogan and Illingworth, 2003), etc. The horizontal displacement due to wind shear also contributes to the decrease of 
$\sigma_{\text {lnwWC }}$ from the cloud top to around $10.5 \mathrm{~km}$. Sample 1 is a geometrically thick cirrus event. Accordingly, the small-scale variability due to sublimation usually occurs near the cloud base (Hogan and Illingworth, 2003), leaving an increase of $\sigma_{\ln I W C}$ within $1 \mathrm{~km}$ near cloud base. For Sample 2, the homogenization of the IWC structure due to wind shear also exists. Accordingly, the slope of the power spectra varies with height in a way similar to that of Sample 1. As indicated by Fig. 1, small-scale structures are apparent for Sample 1 near the cloud top. The small-scale structures contribute to statistical noise, which fails the curve regression procedure expressed by Equation (4). In this case, the slope is relaxed to $-5 / 3$. Within $1 \mathrm{~km}$ of the cloud base, the $\sigma_{\text {lnIWC }}$ larger than 1.5 is replaced by 1.5 to guarantee that the number concentration of the IWC conforms to the assumed lognormal distribution (Hogan and Kew, 2005).

Zinner and Mayer (2006) suggest that the 3-D cloud fields should be gridded at a spatial resolution smaller than the free path length to better quantify the 3-D radiative effects. The free path length $(l)$ is determined by Equation (5) (Davis et al. 1997):

$$
l=[(1-g) \sigma]^{-1}
$$

where $g$ denotes the asymmetry parameter, and $\sigma$ the extinction coefficient, which is determined by the IWC and effective diameter (De). The focus of this study is COT rather than De. Therefore, De is set to a constant value $(60 \mu \mathrm{m})$ throughout the cirrus clouds. Kalesse (2009) also adopted a similar simplification.

For the generated 3-D cirrus clouds, $l$ is approximately $500 \mathrm{~m}$ and $250 \mathrm{~m}$ for Sample 1 and Sample 2, respectively. However, the 3-D radiative transfer process is performed with the same resolution $(250 \mathrm{~m})$ to discard the differences in spatial resolution of the radiative transfer simulations. Two sets of cirrus fields corresponding to Sample 1 and Sample 2 are generated by Cloudgen based on the input parameters presented by Fig. 2. The cirrus clouds cover a $15 \mathrm{~km} \times 15 \mathrm{~km}$ region gridded at a $250 \mathrm{~m} \times 250 \mathrm{~m} \times 150 \mathrm{~m}$ resolution. Accordingly, there are $60 \times 60$ subpixels of a cloud scene for each cloud field. Fig. 4 shows a typical example corresponding to the two samples. 

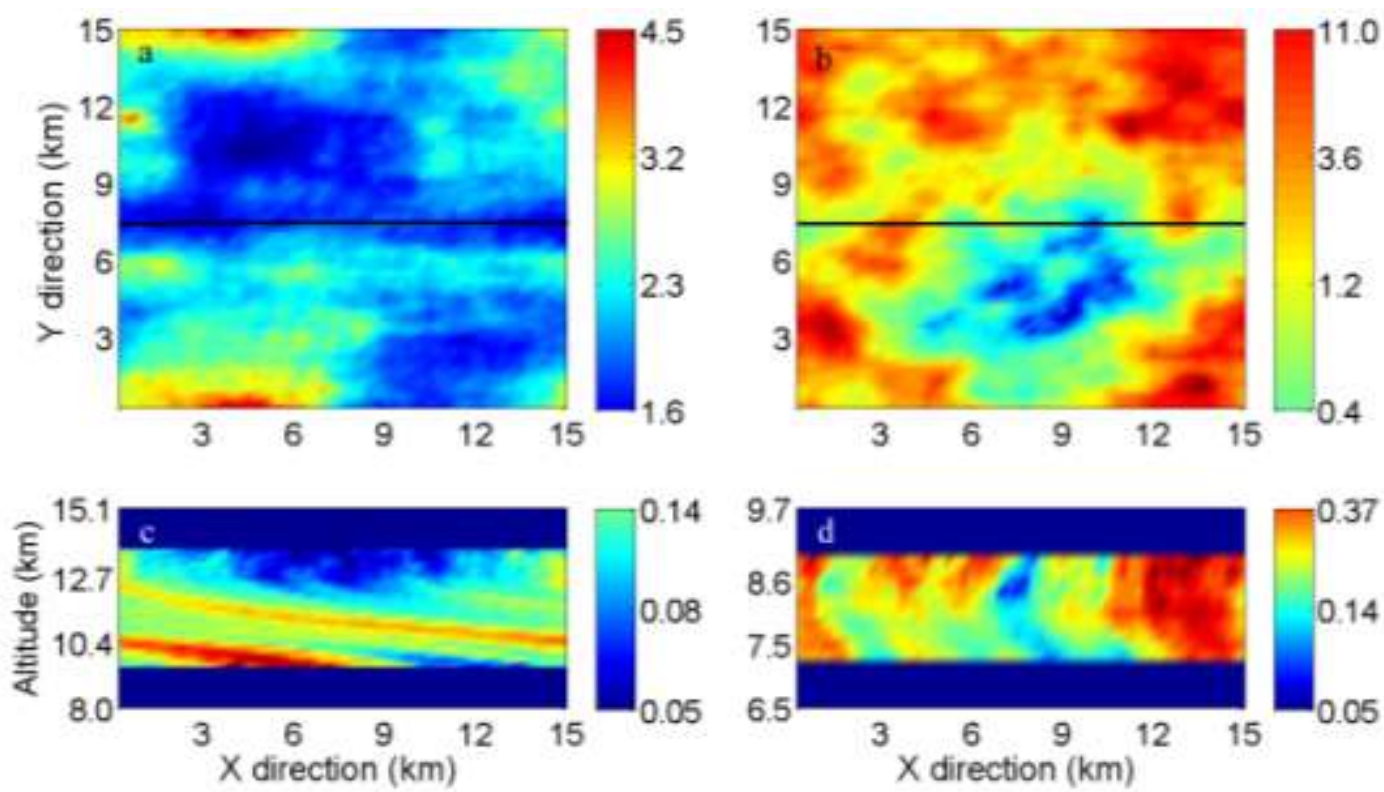

Fig.4 a: $15 \mathrm{~km} \times 15 \mathrm{~km}$ COT simulated at $0.86 \mu \mathrm{m}$ with a horizontal spatial resolution of $250 \mathrm{~m}$ for Sample $1 . \mathrm{b}: 15$ $\mathrm{km} \times 15 \mathrm{~km}$ COT simulated at $0.86 \mu \mathrm{m}$ with a horizontal spatial resolution of $250 \mathrm{~m}$ for Sample 2. c: the $\mathrm{x}-\mathrm{z}$ view of the ice water content $\left(\mathrm{g} / \mathrm{m}^{3}\right)$ through the black line shown in a. $\mathrm{d}$ : the $\mathrm{x}-\mathrm{z}$ view of the ice water content $\left(\mathrm{g} / \mathrm{m}^{3}\right)$ through the black line shown in $b$.

Table 2 summarizes the mean optical thickness $(\overline{C O T})$, the mean extinction coefficient $\left(\overline{k_{e x t}}\right)$, the inhomogeneity parameter $(\xi)$, and the resolution. $\xi$ is defined as the ratio of the standard deviation of COT to $\overline{C O T}$ (Szczap et al. 2014). The resolution denotes the spatial resolution of the radiative transfer simulation. It is noted that the cloud coverage of the two samples is 100 percent.

Table 2 The mean cloud optical properties of the two samples.

\begin{tabular}{ccccc}
\hline Cirrus Sample & $\overline{C O T}$ & $\overline{k_{\text {ext }}}\left(\mathrm{km}^{-1}\right)$ & $\xi$ & Resolution $(\mathrm{m})$ \\
\hline Sample 1 & 2.2 & 0.5427 & 0.21 & 250 \\
Sample 2 & 2.9 & 2.2155 & 1.24 & 250 \\
\hline
\end{tabular}

\section{Methodology}

\subsection{Experiment design}

The Top-of-Atmosphere (TOA) reflectance is simulated by the Spherical Harmonic Discrete Ordinate Method (SHDOM) radiative transfer model. The SHDOM is a 3-D radiative transfer model capable of simulating the radiative transfer process both at IPA and 3-D modes. For the IPA mode, independent pixels are treated as separate periodic domains (Evans, 1998), and an exactly corresponding relationship exists between the reflectance and COT for a selected pixel. The input parameters of SHDOM are specified at 3-D 
grid points, and the output parameter is the radiance at specified directions and altitudes. Pincus and Evans (2009) conducted an intercomparison between SHDOM and a community Monte Carlo model and suggested the comparative accuracy of the two models in simulating pixel-by-pixel intensity but with higher efficiency for SHDOM.

The COT is retrieved for two sensor settings: MODIS and SEVIRI. Two bands are chosen with one a VIS band $(0.86 \mu \mathrm{m}$ band for MODIS and $0.64 \mu \mathrm{m}$ band for SEVIRI) and the other a NIR band $(2.13 \mu \mathrm{m}$ band for MODIS and $1.64 \mu \mathrm{m}$ band for SEVIRI). The reflectivity is obtained at a horizontal resolution of $1 \mathrm{~km}$ for MODIS and $2 \mathrm{~km}$ for SEVIRI. The calculations are performed according to the steps listed below.

1. Eight 3-D cirrus clouds corresponding to either of the two cirrus samples are generated by Cloudgen. According to Section 2.4, the cirrus clouds are gridded at $250 \mathrm{~m} \times 250 \mathrm{~m} \times 150 \mathrm{~m}$ resolution. For a certain cirrus sample, the generated cirrus clouds are statistically equivalent. Their only differences lie in the way by which IWC is stochastically distributed.

2. The TOA reflectance of the generated cirrus clouds is simulated for the solar zenith angles of $30^{\circ}$ and $60^{\circ}$, solar azimuth angle of $0^{\circ}$ and viewing zenith angle of $0^{\circ}$ (nadir observation). The radiative transfer process is simulated both at the IPA and 3-D modes.

3. The reflectance of the subpixels is fused into the reflectance of pixels corresponding to the horizontal resolution of the two sensors. The fusion is performed by the averaging of 16 and 64 subpixels of the SHDOM simulation for MODIS and SEVIRI.

4. The COT and De are retrieved by the reflectance of pixels corresponding to the sensors' resolution, and the retrieval errors are quantified. The retrieval algorithm and the error analyzing methods are introduced by Section 3.2.

5. In step 2, the solar azimuth angle is reset to $45^{\circ}, 90^{\circ}$ and $180^{\circ}$. Steps 3 and 4 are repeated.

6. Two additional cirrus clouds are generated in a similar way to that of Step 1, with each of them corresponding to Sample 1 and Sample 2. The two cirrus clouds are generated for validation purposes.

\subsection{Cloud optical properties retrieval algorithm}

The COT and De are retrieved by the Look-Up Table (LUT) approach (Platnick et al. 2015). To maintain consistency in the radiative transfer simulation, the LUT is also constructed by the SHDOM for plane-parallel cloud fields with a set of predefined conditions: 1) Solar zenith angle is set to $30^{\circ}$ and $\left.60^{\circ}, 2\right)$ De increases from $10 \mu \mathrm{m}$ to $120 \mu \mathrm{m}$ with an increment of $10 \mu \mathrm{m}$, and 3) COT is set to a non-interval series which is 0.01 , 0.2 , 0.4, 0.6, 0.8, 1.0, 1.5, 2.0, 3.0, 4.0, 5.0, 6.0, 7.0, 8.0, 9.0, 10.0, and 15.0. Fig. 5 shows a typical example of the LUT for the MODIS and SEVIRI sensor settings. 


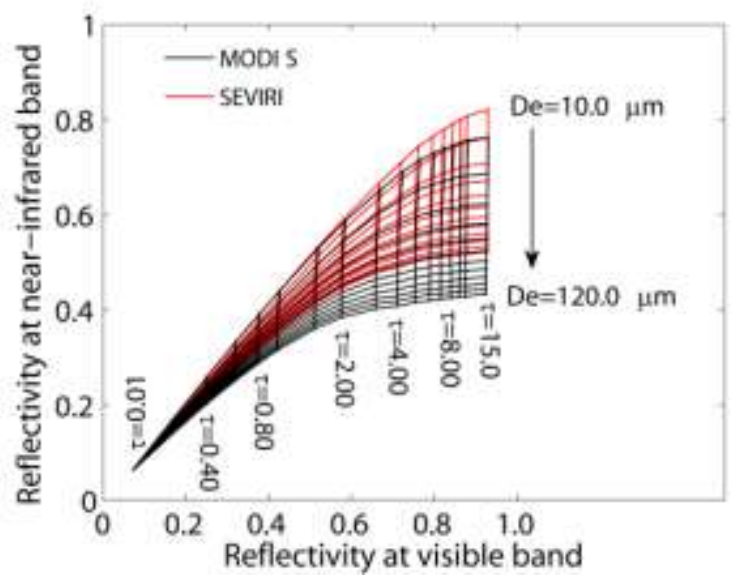

Fig. 5 SHDOM simulated nadir reflectivity for MODIS and SEVIRI for plane-parallel cirrus clouds comprising values of optical thickness $0 \leq \mathrm{T} \leq 15$ and effective diameter $10 \leq \mathrm{De} \leq 120 \mu \mathrm{m}$ with a solar zenith angle of $30^{\circ}$.

Davis et al (1997) is among the first to calculate the errors on the retrieved COT for 3-D cloud fields. Their basic idea of calculating the retrieval errors is adopted by Zinner and Mayer (2006), who further separated the retrieval errors into errors due to PPA and IPA. For COT, the relative error, the relative error due to PPA, and the relative error due to IPA are represented by $\delta \tau, \delta \tau_{P P A}$ and $\delta \tau_{I P A}$. By simple deduction, these three parameters are calculated by Equations $(6) \sim(8)$ in this study.

$$
\begin{gathered}
\delta \tau=\left[f\left(\overline{R_{3-D}}\right)-\overline{f\left(R_{I P A}\right)}\right] / \overline{f\left(R_{I P A}\right)} \\
\delta \tau_{P P A}=\left[f\left(\overline{R_{I P A}}\right)-\overline{f\left(R_{I P A}\right)}\right] / \overline{f\left(R_{I P A}\right)} \\
\delta \tau_{I P A}=\left[f\left(\overline{R_{3-D}}\right)-f\left(\overline{R_{I P A}}\right)\right] / \overline{f\left(R_{I P A}\right)}
\end{gathered}
$$

where $f$ denotes the reflectance-COT function shown by Fig. 5. The $R_{I P A}$ and $R_{3-D}$ denote the reflectance of a certain subpixel simulated at the IPA and 3-D modes. The $\overline{R_{I P A}}$ and $\overline{R_{3-D}}$ denote the averaged reflectivity at the pixel-level resolution for either mode. The $\overline{f\left(R_{I P A}\right)}$ denotes the pixel-averaged COT, which is calculated by averaging the COT of the subpixels within a pixel. Because an exactly corresponding relationship exists between the reflectance and COT at IPA mode, $\overline{f\left(R_{I P A}\right)}$ represents the real COT. The relative error is calculated by dividing the absolute error by $\overline{f\left(R_{I P A}\right)}$. A similar method is adopted in calculating the retrieval errors on De, which are computed and presented in absolute values.

In order to quantify the relative contribution of the 3-D radiative effects and horizontal heterogeneity effects to $\delta \tau$, we define a parameter denoted by $\eta(I P A)$ :

$$
\eta(I P A)=\left|\delta \tau_{I P A}\right| /\left(\delta \tau_{P P A}|+| \delta \tau_{I P A} \mid\right)
$$




\section{Results}

\subsection{COT Error Analyses}

\subsubsection{Consistency check}

Fig. 6 illustrates the consistency check of the LUT approach. COT is retrieved from the reflectance simulated at the IPA mode. The horizontal resolution is set to the subscale $(250 \mathrm{~m})$. In this case, no PPA errors and IPA errors should be expected. As indicated, the retrieved COT ( $\mathrm{COT}^{\mathrm{ret}}$ ) agrees well with the real COT. The correlation coefficient is 0.99 ; the slope and the intercept are 0.98 and 0.04 , respectively. Further analyses indicate a Gaussian PDF symmetric to 0 . The tail of the PDF on either side is within $-5.0 \%-5.0 \%$ (the embedded plot in Fig. 6). The errors are small, and are mainly caused by the LUT approach (linear interpolation) and SHDOM model simulations (truncation errors). The consistency check is performed for Sample 1 with MODIS sensor settings. The solar azimuth angle and solar zenith angle are set to $0^{\circ}$ and $30^{\circ}$, respectively. There also exists good consistency between the real and retrieved COT for SEVIRI sensor settings and other sun-view geometries (not shown in Fig. 6). Therefore, the LUT approach is deemed accurate.

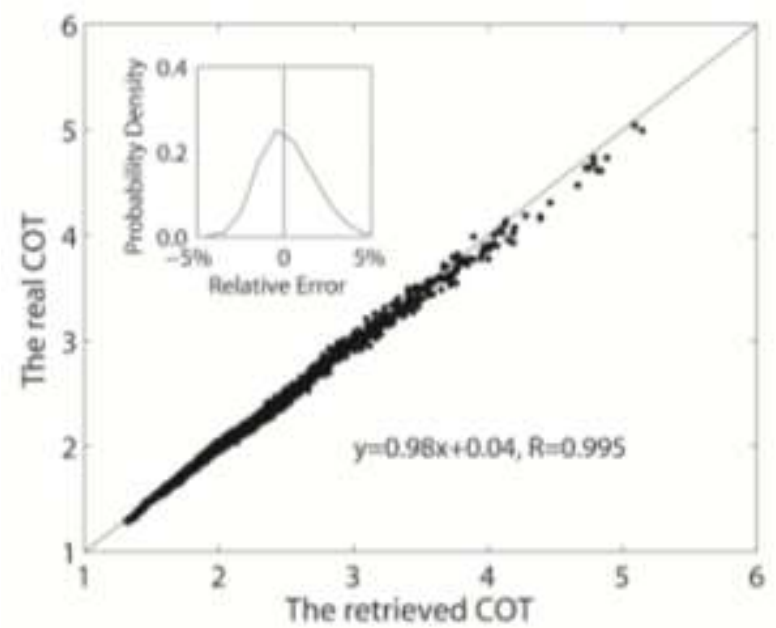

Fig. 6 Comparison of the $C O T^{\text {ret }}$ and the real COT for sample 1 with MODIS sensor settings. The solar azimuth angle and solar zenith angle are set to $0^{\circ}$ and $30^{\circ}$, respectively.

\subsubsection{Dependence on solar zenith angle and resolution}

Fig. 7 shows the PDFs of the relative errors on $C O T^{r e t}$. It is apparent that the tails of the PDF are extended on either side with an increase of the solar zenith angle. Besides, the PDFs are skewed, especially for the larger solar zenith angle, implying that the PDFs should be analyzed on each side separately. 

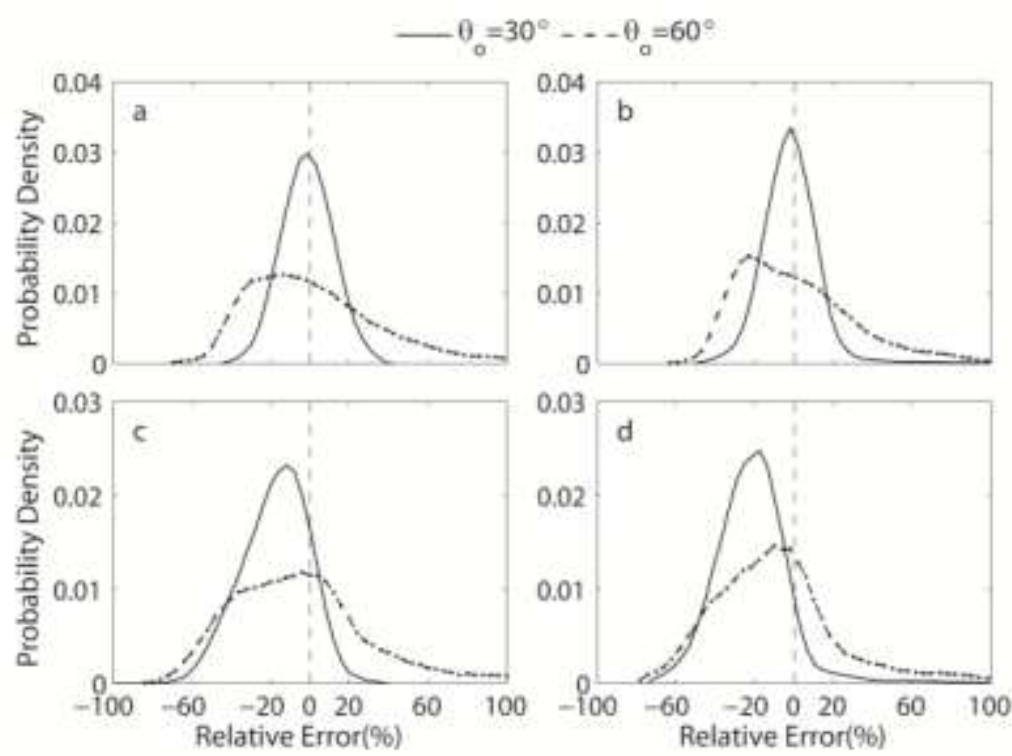

Fig.7 The PDFs of the relative errors on the $C O T^{\text {ret }}$. a denotes the results for Sample 1 with MODIS sensor settings. b denotes the results for Sample 1 with SEVIRI sensor settings. $c$ denotes the results for Sample 2 with MODIS sensor settings. $d$ denotes the results for Sample 2 with SEVIRI sensor settings. $\theta_{0}$ denotes the solar zenith angle.

We introduce the upper bound $\left(\delta \tau_{\text {sup }}\right)$ and lower bound $\left(\delta \tau_{\text {inf }}\right)$ defined as the threshold value outside which the probability accounts for $16 \%$ on either side. A randomly selected relative error lies inside the region spanned by the $\delta \tau_{\text {sup }}$ and $\delta \tau_{\text {inf }}$ with a $68 \%$ probability (confidence interval). Therefore, $\delta \tau_{\text {sup }}$ and $\delta \tau_{\text {inf }}$ are collectively called the error bounds. If $\delta \tau_{\text {inf }}$ equals $\delta \tau_{\text {sup }}$, the domain-averaged relative error on $C O T^{\text {ret }}$ would be an exact value without uncertainty. Therefore, the uncertainty of the relative error is defined by Equation (10).

$$
\Delta \tau=\left(\tau_{\text {sup }}-\tau_{\text {inf }}\right) / 2
$$

where the term $\Delta \tau$ denotes the uncertainty.

The confidence interval is set to $68 \%$ to maintain consistency with previous research (Immler et al. 2010; Glantz and Tesche, 2012) where the retrieval uncertainty is set to the standard deviation of the selected parameter that conforms to a Gaussian distribution. In such case, a randomly selected parameter lies inside the region which centers at the mean value and extends on the left and right sides by the magnitude quantified by the uncertainty with a $68 \%$ probability.

Table 3 summarizes the statistics of relative errors on the retrieved COT. $\overline{\delta \tau_{I P A}}$ and $\overline{\delta \tau_{P P A}}$ denote the domain-averaged relative errors due to IPA and PPA, respectively. The term $\overline{\delta \tau}$ denotes the domain-averaged relative error (the sum of $\overline{\delta \tau_{I P A}}$ and $\overline{\delta \tau_{P P A}}$ ). The terms $\eta(I P A)$ and $\Delta \tau$ denote the parameter defined by Equation (9) and the retrieval uncertainty calculated by Equation (10). As indicated, the 
dependence of the statistics of the retrieval errors of COT on the horizontal resolution and solar zenith angle is evident. First, $\overline{\delta \tau}$ is smaller for SEVIRI than MODIS. The COT is much more sensitive to reflectance at VIS bands than NIR bands. The optical properties of the SRAC ice cloud spectral model exhibit no distinct differences at the VIS bands for the two sensors, leaving nearly identical reflectance, which is also validated in Fig. 5. Therefore, the smaller $\overline{\delta \tau}$ for SEVIRI could be attributed to the coarser resolution. One main reason is that the increase of the pixel size will directly decrease $\overline{\delta \tau_{P P A}}$. Second, $\overline{\delta \tau}$ and $\Delta \tau$ increase as solar zenith angle increase from $30^{\circ}$ to $60^{\circ}$. On the domain average, the COT is underestimated for solar zenith angle of $30^{\circ}$. With the increase of solar zenith angle, $\overline{\delta \tau}$ even turns into positive values for Sample 1. Meanwhile, $\eta(I P A)$ is increased as well, implying that the contribution of the 3-D radiative effects to $\overline{\delta \tau}$ becomes much more important for the larger solar zenith angle.

Table 3 The statistics of the relative errors on the COT $^{\text {ret }}(\%)$

\begin{tabular}{|c|c|c|c|c|c|c|c|c|}
\hline \multirow{3}{*}{$\begin{array}{c}\text { Cirrus Event } \\
\text { Payload } \\
\text { Solar zenith }\end{array}$} & \multicolumn{4}{|c|}{ Sample 1} & \multicolumn{4}{|c|}{ Sample 2} \\
\hline & \multicolumn{2}{|c|}{ MODIS } & \multicolumn{2}{|c|}{ SEVIRI } & \multicolumn{2}{|c|}{ MODIS } & \multicolumn{2}{|c|}{ SEVIRI } \\
\hline & $30^{\circ}$ & $60^{\circ}$ & $30^{\circ}$ & $60^{\circ}$ & $30^{\circ}$ & $60^{\circ}$ & $30^{\circ}$ & $60^{\circ}$ \\
\hline$\overline{\delta \tau}$ & -1.0 & 3.3 & -1.5 & 1.4 & -17.1 & -5.1 & -24.1 & -11.6 \\
\hline$\overline{\delta \tau_{I P A}}$ & -0.8 & 3.4 & -1.0 & 1.8 & -11.9 & 0 & -11.8 & 0.8 \\
\hline$\overline{\delta \tau_{P P A}}$ & -0.2 & -0.1 & -0.5 & -0.4 & -5.2 & -5.1 & -12.3 & -12.4 \\
\hline$\eta(I P A)$ & 94.8 & 97.6 & 90.7 & 96.5 & 72.1 & 80.3 & 53.2 & 62.2 \\
\hline$\Delta \tau$ & 10.0 & 27.5 & 7.5 & 27.5 & 12.5 & 20.0 & 12.5 & 20.0 \\
\hline
\end{tabular}

For small solar zenith angles, the cirrus cloud is mainly illuminated by the solar direct beam from the top. In this case, the radiation tends to be channeled from the optically thicker regions to the optically thinner regions. On the domain average, the reflectance of the optically thicker regions tends to be decreased (underestimation of $\mathrm{COT}$ ), while the reflectance for the optically thinner regions tends to be increased (overestimation of COT). This mechanism is termed the channeling effect (Davis and Marshak, 2001; Várnai and Marshak, 2003; Zinner and Mayer, 2006). With the increase of the solar zenith angle, the studied region is illuminated by the solar beam from cloud top and side illumination from adjacent regions. If the studied region is optically thicker than the surrounding regions, the incident radiance tends to be increased compared with that of the IPA mode because of less attenuation from the side, leaving an increase of reflectance for the studied region and an overestimation of COT. Such a mechanism is termed the side illumination effect (Várnai and Marshak, 2003). In contrast, the reflectance of the optically thinner regions is reduced by the optically thicker regions by a mechanism similar to the shading effects in bumpy clouds (Zinner and Mayer, 2006). In summary, the underestimation is caused by the PPA, the channeling effects (for optically thicker regions) and 
the shading effects (for optically thinner regions), while potential contributing causes to the overestimation include the channeling effects (for optically thinner regions) and the side illumination effects (for optically thicker regions). In real cases involving the mechanisms mentioned above, it is difficult to trace the errors to a specific mechanism. Nevertheless, we can see the extent to which their combined effects affect the remote sensing of the COT. As indicated in Fig. 5, the relationship between the reflectance and COT is a monotonically increasing concave function. Therefore, the reflectance change of the same magnitude has stronger effects on the remote sensing of COT for the optically thicker regions than the optically thinner regions, which is a potential explanation to the farther extension of the PDF's tail on the right side for the solar zenith angle of $60^{\circ}$ (Fig. 7).

\subsubsection{Dependence on solar azimuth angle}

Várnai and Marshak (2003) indicated that the distribution of cloud fields in along-track and cross-track directions relative to the solar direct beam influences the 3-D radiative effects by different extents. Therefore, errors on the $C O T^{\text {ret }}$ should have a dependence on the solar azimuth angle.

The retrieval process is performed by Step 5 in Section 3.1, and the results are shown in Fig. 8. For comparison, the results for solar azimuth angle of $0^{\circ}$ are also presented. The domain-averaged relative error and uncertainty vary with solar zenith angle and resolution with the characteristics similar to those for solar azimuth angle of $0^{\circ}$. Namely, the domain-averaged relative error increases with increasing solar zenith angle and decreases with coarsening resolution. With the solar zenith angle increasing from $30^{\circ}$ to $60^{\circ}$, the uncertainty is increased, which is also validated by the decrease of the lower bounds and the increase of the upper bounds. The comparison between different solar azimuth angles indicates that the domain-averaged relative error remains nearly constant for different solar azimuth angles, with the maximum variation confined below $5 \%$. Meanwhile, the maximum variation of uncertainty with solar azimuth angle reaches up to $10 \%$. The upper and lower bounds change with solar zenith angle by different extents for different solar azimuth angles, implying that the influences of the 3-D radiative effects do have a dependence on the solar azimuth angle. However, the influences of the solar azimuth angle on the remote sensing of COT are less discernible than in previous research (Zinner and Mayer, 2006; Kato and Marshak, 2009). The main cause for such a phenomenon lies in the inner structure of the two selected cirrus samples that are rather flat and fully covered. The external heterogeneity due to bumps and gaps is the most predominant factor affecting the 3-D radiative effects (Dim et al. 2007). For the studied cirrus clouds, the external heterogeneity effects are trivial. 

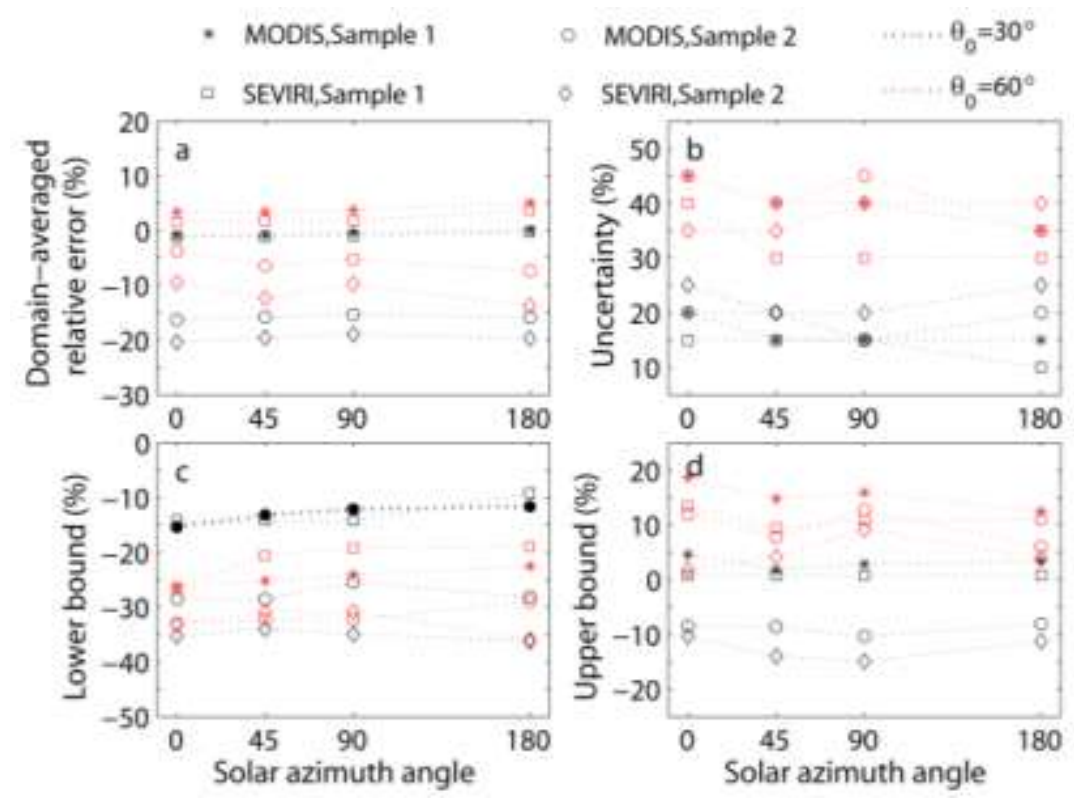

Fig. 8 The dependence of the domain-averaged relative errors, uncertainties, upper bounds, and lower bounds on the solar azimuth angle (cf. Fig. 7)

\subsubsection{Parameterization of relative error and error bounds}

Fig. 9 presents the dependence of the bin-averaged relative error on the $C O T^{\text {ret }}$. The bin-averaged relative errors are calculated as the averaged values of the relative errors for the discrete $C O T^{\text {ret }}$ bins with an increment of 0.5 . During the data processing procedure, intervals with the number of valid COT $^{\text {ret }}$ samples less than 64 are screened out to guarantee the representativeness of the statistical results. As indicated, the relationships of the bin-averaged relative errors and $C O T^{\text {ret }}$ are complicated for the solar zenith angle of $30^{\circ}$. Several mechanisms influence the 3-D radiative transfer process, and their blending effects on the remote sensing process are rather complicated. Therefore, the reasons underlying such phenomena remain uncertain. Nevertheless, the bin-averaged relative errors could be fitted to linear functions by the $C O T^{r e t}$ for the solar zenith angle of $60^{\circ}$ with the regression coefficients larger than 0.96 . The slopes and intercepts of the linear regressions are positive and negative values, implying that the COT tends to be overestimated for large $C O T^{\text {ret }}$ and underestimated for small $C O T^{\text {ret }}$. The linear regression between the bin-averaged relative error and the $C O T^{r e t}$ provides a basis for the correction of the $C O T^{r e t}$. The analyses below are only applicable to the solar zenith angle of $60^{\circ}$ unless otherwise mentioned. 

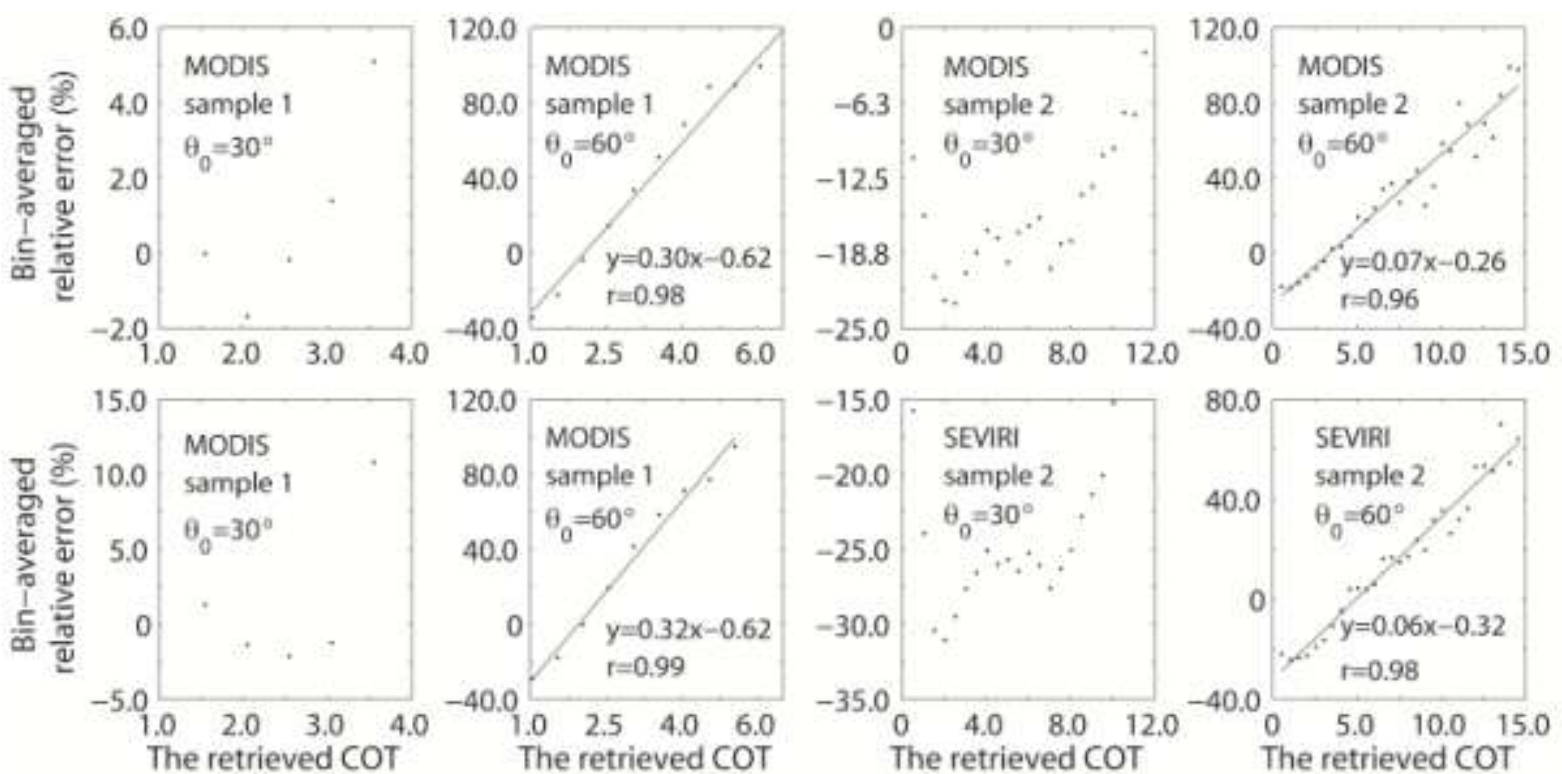

Fig. 9 The dependence of the bin-averaged relative errors on the $\operatorname{COT}^{\text {ret }} . \theta_{0}$ denotes the solar zenith angle. $r$ denotes the regression coefficient.

By making a simple deduction of linear functions, we introduce a $C O T^{\text {ret }}$ correction method:

$$
\mathrm{COT}^{\text {ret, }}=\frac{\mathrm{COT}^{\text {ret }}}{a C O T^{\text {ret }}+b+1}
$$

where $a$ and $b$ denote the slope and intercept of linear regression, respectively, shown by Fig. 9. The term $C O T^{\text {ret,' }}$ denotes the corrected value of $C O T^{\text {ret }}$.

To validate the accuracy of the correction method, the COT is retrieved for two other cirrus clouds following Step 6 in Section 3.1. The results indicate that the domain-averaged relative error of COT $^{\text {ret,' }}$ ranges from $-2.3 \%$ to $1.5 \%$ (Fig. 10), far less than that of the $C O T^{\text {ret }}$. Therefore, the correction method expressed by Equation (11) is deemed effective.
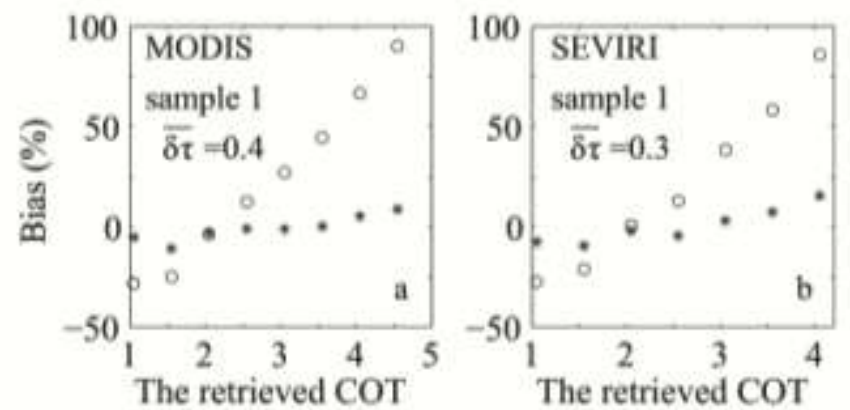

The retrieved COT
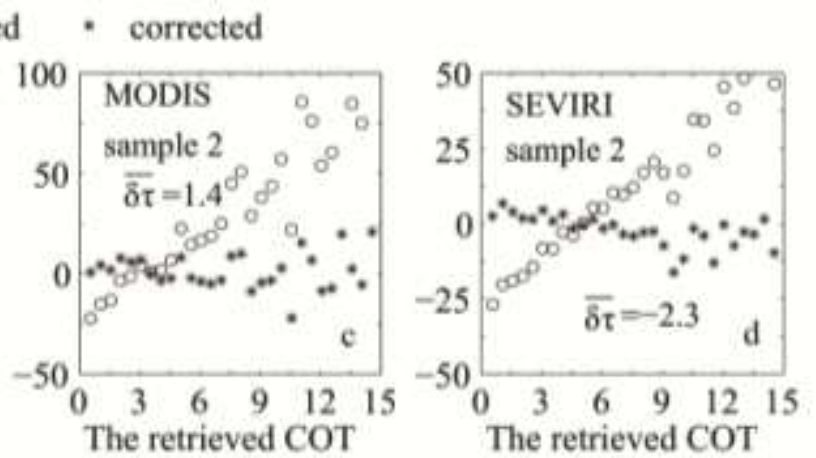

Fig. 10 Comparison of the bin-averaged relative errors of the $C O T^{r e t^{\prime}}$ and the $C O T^{r e t} \cdot \overline{\delta \tau}$ denotes the domain-averaged relative error of the $C O T^{\text {ret, }}$

Similarly, the error bounds could be fitted to linear functions. For a randomly selected $C O T^{\text {ret }}$, the 
corresponding relative error is expected to lie inside the range spanned by the upper and lower bounds. The effectiveness of the error bounds is validated by the two cirrus clouds generated by Step 6 , and the results are presented in Fig. 11. As indicated, for larger $C O T^{\text {ret }}$ values, there will be larger ranges spanned by the error bounds. Such a result implies an increase of uncertainty with increasing $C O T^{\text {ret }}$, and vice versa. In Fig. 11, the hit rate is calculated as the ratio of the number of valid pixels, which denotes the pixels with relative error lying inside the error bounds, to the total number of pixels. As indicated, the hit rate is larger than $60 \%$.

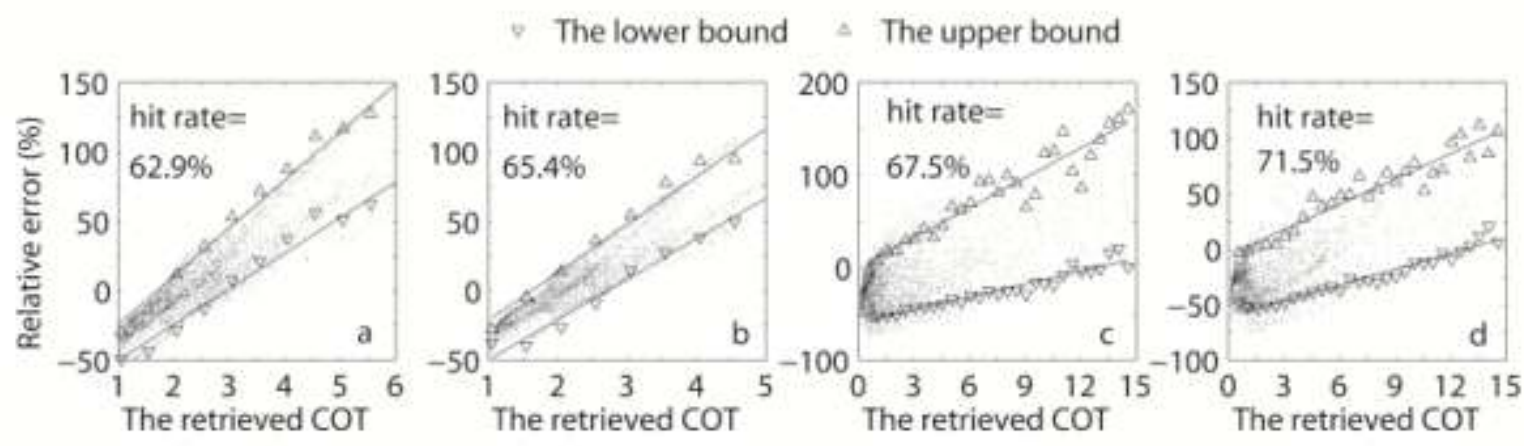

Fig. 11 The upper bounds, lower bounds, and the retrieval errors on the $C O T^{\text {ret }}$. The scatters denote the retrieval errors on the $C O T^{\text {ret }}$ (cf. Fig. 10)

The linear dependence of the domain-averaged relative errors and error bounds for the $C O T^{\text {ret }}$ is also applicable to the solar zenith angles of $45^{\circ}, 90^{\circ}$ and $180^{\circ}$, but a certain tuning of slopes and intercepts of linear regression is necessary. The domain-averaged relative errors on the corrected $C O T^{\text {ret }}$ and the hit rates are summarized in Table 4. For comparison, the results for solar azimuth angle of $0^{\circ}$ are also presented. It is apparent that the absolute values of the domain-averaged relative errors are confined below $5 \%$. In addition, the hit rates vary from $62.9 \%$ to $87.9 \%$, implying the accuracy of the estimated uncertainty.

Table 4 The domain-averaged relative errors (after correction) and the hit rates

\begin{tabular}{ccccccc}
\hline \multirow{2}{*}{$\begin{array}{c}\text { Cirrus Event } \\
\text { Payload }\end{array}$} & Solar azimuth angle & \multicolumn{2}{c}{ Sample 1 } & & \multicolumn{2}{c}{ Sample 2 } \\
\cline { 3 - 4 } \cline { 6 - 7 } & $0^{\circ}$ & MODIS & SEVIRI & & MODIS & SEVIRI \\
Domain-averaged & $45^{\circ}$ & 0.4 & 0.3 & & 1.4 & -2.3 \\
relative error (\%) & $90^{\circ}$ & -0.9 & -2.8 & & -0.4 & -1.7 \\
& $180^{\circ}$ & 1.8 & 0.3 & & 3.8 & -4.3 \\
& $0^{\circ}$ & 2.5 & 2.0 & & -0.5 & -4.6 \\
Hit Rate (\%) & $45^{\circ}$ & 62.9 & 65.4 & & 67.5 & 71.5 \\
& $90^{\circ}$ & 70.0 & 72.5 & & 80.6 & 82.3 \\
& $180^{\circ}$ & 77.4 & 79.7 & & 74.1 & 78.8 \\
& & 87.9 & 87.9 & & 83.5 & 87.3 \\
\hline
\end{tabular}




\subsection{De error analysis}

\subsubsection{Consistency check}

Fig. 12 shows the results for the consistency check of the retrieved De (denoted by $D e^{r e t}$ ). At IPA mode, the PDF of the error on the $D e^{\text {ret }}(\delta D e)$ conforms to a Gaussian function. The mean and standard deviation are $0.2 \mu \mathrm{m}$ and $3.5 \mu \mathrm{m}$, respectively. The Gaussian PDF also applies to other sensor settings and sun-view geometries (not shown in the Fig. 12), where the mean (absolute value) and standard deviation of $\delta D e$ are confined below $2.0 \mu \mathrm{m}$ and $5.0 \mu \mathrm{m}$ respectively. Therefore, the LUT approach is deemed accurate in the remote sensing of De.

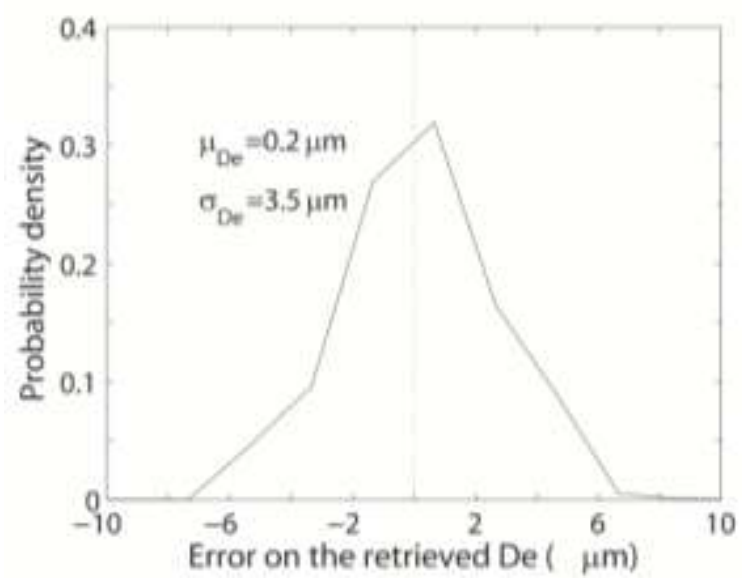

Fig. 12 Comparison of the retrieved and the real De for sample 1 with MODIS sensor settings. $\mu_{D e}$ and $\sigma_{D e}$ denote the mean and standard deviation of the $\delta D e$. The solar azimuth angle and solar zenith angle are set to $0^{\circ}$ and $30^{\circ}$.

\subsubsection{De error analyses}

The PDFs of $\delta D e$ are presented in Fig. 13. As indicated, the PDFs become stockier with the increase of the solar zenith angle, which is similar to the results for the COT except that the PDFs of $\delta D e$ are approximately symmetric. Therefore, the PDFs could be fitted to Gaussian functions. Similar characteristics of the PDFs for the solar azimuth angles of $45^{\circ}, 90^{\circ}$ and $180^{\circ}$ also exist. In order to be concise, they are not presented in Fig. 13. 

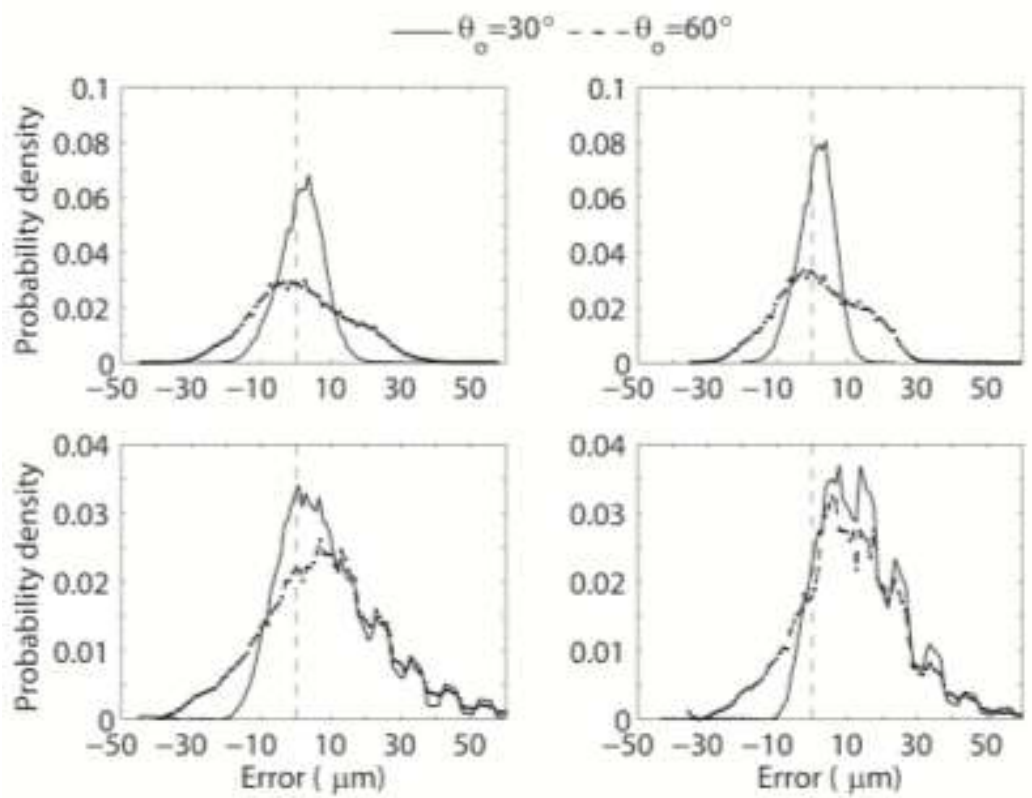

Fig.13 The PDFs of $\delta D e$ (cf. Fig. 7)

The domain-averaged errors and uncertainties of $\delta D e$ are summarized in Table 4. As mentioned above, the PDFs of the $\delta D e$ conform to Gaussian functions. Therefore, the uncertainties are calculated as the standard deviation of $\delta D e$. Similar to the results for the $C O T^{r e t}$, the uncertainties increase with lower solar elevation, which is directly related to the roughening of the radiation fields. In addition, De is overestimated on the domain average, which agrees well with results by Kalesse (2009). For Sample 1, variation of the domain-averaged errors with solar zenith angle is not as discernible as that for Sample 2 and is almost negligible. With solar zenith angle increasing from $30^{\circ}$ to $60^{\circ}$, the PDFs are extended on either side by approximately the same extent ( $a$ and $b$ in Fig. 13). For Sample 2, the domain-averaged errors degrade with increasing solar zenith angle, and the PDFs are preferably extended on the left side ( $c$ and $d$ in Fig. 13). As indicated in Table 2, Sample 2 is optically thicker and more heterogeneous than Sample 1, and the influences by the 3-D radiative effects and horizontal heterogeneity effects are distinctly different for the two cirrus samples. Therefore, the differences in the variation of the domain-averaged errors with the solar zenith angle between Sample 1 and Sample 2 are rational. 
Table 4 The domain-averaged relative errors (after correction) and the hit rates

\begin{tabular}{ccccccc}
\hline \multirow{2}{*}{$\begin{array}{c}\text { Cirrus Event } \\
\text { Payload }\end{array}$} & Solar azimuth angle & \multicolumn{2}{c}{ Sample 1} & & \multicolumn{2}{c}{ Sample 2 } \\
\cline { 3 - 4 } \cline { 6 - 7 } & & MODIS & SEVIRI & & MODIS & SEVIRI \\
Domain-averaged & $0^{\circ}$ & 0.4 & 0.3 & & 1.4 & -2.3 \\
relative error (\%) & $45^{\circ}$ & -0.9 & -2.8 & & -0.4 & -1.7 \\
& $90^{\circ}$ & 1.8 & 0.3 & & 3.8 & -4.3 \\
& $180^{\circ}$ & 2.5 & 2.0 & & -0.5 & -4.6 \\
Hit Rate (\%) & $0^{\circ}$ & 62.9 & 65.4 & & 67.5 & 71.5 \\
& $45^{\circ}$ & 70.0 & 72.5 & & 80.6 & 82.3 \\
& $90^{\circ}$ & 77.4 & 79.7 & & 74.1 & 78.8 \\
& $180^{\circ}$ & 87.9 & 87.9 & & 83.5 & 87.3 \\
\hline
\end{tabular}

Unlike the cases for the COT, the influences of horizontal resolution on the remote sensing of De are not explicitly discussed here. As is indicated by Fig. 5, the reflectance at the NIR band is smaller for the MODIS than for the SEVIRI sensor settings. Zhang et al. (2012) indicated that the 3-D radiative effects affect the effective radius derived from $2.1 \mu \mathrm{m}$ and $3.7 \mu \mathrm{m}$ by different extents. Although the intercomparison between $1.6 \mu \mathrm{m}$ and $2.13 \mu \mathrm{m}$ was not discussed in their study, their results have certain implications for this study. Namely, the differences in the results between MODIS and SEVIRI have their roots in the horizontal resolution and in the optical properties at the NIR bands.

\section{Conclusions}

In this study, the influences of 3-D radiative effects and horizontal heterogeneity effects on the remote sensing of cloud optical thickness (COT) and effective diameter (De) were explored using the SHDOM for cirrus clouds. Two sets of three-dimensional (3-D) stochastic cirrus clouds were generated by the Cloudgen model based on two cirrus events observed by a 35-GHz Millimeter Wave Cloud Radar from the Atmospheric Radiation Measurement (ARM) program. The single scattering properties of ice clouds were derived from the Severely Roughened Aggregated Columns (SRAC) ice cloud spectral model. Errors on the retrieved optical properties were quantified for two cirrus samples (Sample 1 and Sample 2), two solar zenith angles $\left(30^{\circ}\right.$ and $60^{\circ}$ ), four solar azimuth angles $\left(0^{\circ}, 45^{\circ}, 90^{\circ}\right.$, and $\left.180^{\circ}\right)$, and two sensor settings (MODIS and SEVIRI). All the simulations and discussions are for nadir observations. The four main conclusions are summarized below:

1. For the solar zenith angle of $30^{\circ}$, COT is underestimated on the domain average. The domain-averaged relative error varies from $-24.1 \%$ to $-1.0 \%$, depending on the specific cirrus sample and sensor settings. The domain-averaged relative error is smaller for Sample 2, which is optically thicker and more heterogeneous than Sample 1. The result corresponds well with that of Kalesse (2009), who performed the remote sensing of the COT in 3-D cirrus fields for a solar zenith angle of $35^{\circ}$ with nadir observation. 
Similar results were also achieved for a marine stratocumulus case (Kato and Marshak, 2009). For the solar zenith angle of $60^{\circ}$, the domain-averaged relative errors were increased, which confirms the results by Zinner and Mayer (2006) for typical polar-orbiting sensor resolution. The uncertainties increase with lowering solar elevation, with the domain-averaged relative error varying from $-11.6 \%$ to $3.3 \%$. A main cause is the roughening of the radiation fields (Iwabuchi and Hayasaka, 2002). Comparing the results for MODIS and SEVIRI, the dependence of the domain-averaged relative errors on the horizontal resolution was also explored. The domain-averaged relative error is smaller for SEVIRI than for MODIS, showing good consensus with the findings by Várnai and Marshak (2001) where the domain-averaged relative error decreases with coarsening resolution.

2. For the solar zenith angle of $60^{\circ}$, the domain-averaged relative errors and the corresponding uncertainties could be fitted to linear functions by the retrieved COT (COT ${ }^{\text {ret }}$ ). For the smaller $C O T^{\text {ret }}$ values, the relative errors and uncertainties are smaller, and vice versa. Similar results were found by Várnai and Marshak (2001). The linear regression analysis provides a basis to correct $C O T^{\text {ret }}$ and estimate its uncertainty. The accuracy in estimating the uncertainty by the $C O T^{r e t}$ is quantified by the hit rate, which is larger than $60 \%$ in all cases. After correction, the domain-averaged relative error (absolute value) was confined below $2.5 \%$, far less than that before correction.

3. On the domain average, the retrieved $\mathrm{De}\left(D e^{r e t}\right)$ is overestimated by $0.6 \mu \mathrm{m}-15.0 \mu \mathrm{m}$, depending on the specific cirrus sample and sensor settings. The difference in the domain-averaged errors between the two samples is caused by the differences in the internal heterogeneity and optical thickness. The retrieval uncertainties range from $6.7 \mu \mathrm{m}-26.5 \mu \mathrm{m}$ and increase with lowering solar elevation.

4. With the solar azimuth angle varying from $0^{\circ}$ to $45^{\circ}$ to $90^{\circ}$ to $180^{\circ}$, the domain-averaged (relative) errors for the $C O T^{r e t}$ and $D e^{r e t}$ remain almost constant, while the uncertainties show minor deviations from each other. In general, the dependence of the retrieval errors on the solar azimuth angle is not as discernible as that in previous research (Zinner and Mayer, 2006; Kato and Marshak, 2009). The main cause to such a phenomenon is the relatively small external heterogeneity (bumps and gaps) of the two selected cirrus samples. The result implies that the dependence of the 3-D radiative effects on the solar azimuth angle could be properly neglected for cirrus cases with flat tops and full coverage, which simplifies the remote sensing of the cloud optical properties for 3-D cirrus fields.

The results are only valid for the two specific cirrus samples and for the specific resolution of the radiative transfer simulations, and the findings are thought transferable to MODIS-like sensors. However, the influences of the 3-D radiative effects of bumps and gaps on the remote sensing process still remain 
problematic. Therefore, further validation is needed by accounting for more generalized cirrus samples. In addition, there are many practical problems to be solved to produce a viable bias-correction method and uncertainty-estimation method. For example, the parameters determining the linear regression depend on the specific cirrus fields. But the information on 3-D cirrus clouds remains unknown prior to the retrieval process, which poses challenges for the application of the correction and estimation methods. Incorporating a Green's function for a specific cloud formation mechanism, Zinner et al. (2006) iteratively retrieved the 3-D structures of stratocumulus clouds by 2-D high-resolution (approximates to the free path length) measurements. The free path length of cirrus (250 $\mathrm{m}$ in this study) is generally far larger than that of stratocumulus, implying that it may be feasible to perform high-resolution measurements of 2-D radiance fields of cirrus by spaceborne payloads like MODIS. All these problems are the focus of further studies.

\section{Acknowledgements}

This research is supported by National Science Foundation of China (Grant No. 41575020). SHDOM is developed and provided by Professor Evans Franklin from University of Colorado. Cloudgen is developed and provided by Professor Robin Hogan. The continuous microphysical retrieval Value-Added Product and the interpolated sounding Value-Added Product were obtained from the Atmospheric Radiation Measurement (ARM) program. The Severely Roughened Aggregated Columns model was developed and provided by Professor Bryan Baum from SSEC, University of Wisconsin-Madison. Professor Evans Franklin provided much help in running SHDOM radiative transfer model and performing the Wigner-d function expansion of phase function. The authors would like to thank two anonymous reviewers for their thought-provoking suggestions.

\section{Appendix}

The scattering phase function $\left(P_{11}\right)$ of the SRAC ice cloud spectral model is converted to Wigner-d function expansion coefficients by the weighted least squares (WLS) method expressed by Equation (A1).

$$
E(\chi)=\sum_{i=1}^{M} \omega_{i}^{2}\left[P_{11}\left(\Theta_{i}\right)-P_{11}^{*}\left(\Theta_{i}\right)\right]^{2}
$$

where $E(\chi)$ is the residual, $P_{1 I}\left(\Theta_{i}\right)$ is the original phase function, and $\omega_{i}$ is the weight for $\Theta_{i}$, which denotes the scattering angle. The parameter $M$ denotes the total number of Gauss numerical integration nodes. The term $P_{11}^{*}$ is the phase function derived from Wigner-d functions by Equation (A2).

$$
P_{11}^{*}\left(\Theta_{i}\right)=\sum_{k=0}^{N} \sqrt{\frac{2 k+1}{2}} d_{00}^{k}\left(\cos \Theta_{i}\right) \chi_{k}
$$


where $d_{00}^{k}$ is the value of k-order Wigner-d function, $\chi_{k}$ denotes the corresponding expansion coefficient, and $N$ is the order of the Wigner d-function series of the phase function. In the present study, $\mathrm{M}$ and $\mathrm{N}$ are set to 4000 and 1000 , respectively.

$\omega_{i}$ is separated into a mathematical part $\left(\omega^{M}\right)$ and a physical part $\left(\omega^{P}\right) . \omega^{M}$ is designed to guarantee that residuals for different scattering angles are equally important to eliminate extremely large residuals caused by the peak value of the original phase function. Therefore, the mathematical weight is defined by Equation (A3)

$$
\omega_{i}^{M}=1 /\left[P_{11}^{*}\left(\Theta_{i}\right)\right]^{a}
$$

where $a$ is the tuning factor and ranges from 0 to 1 .

By definition,

$$
\int_{0}^{2 \pi} \int_{-1}^{1} P_{11}(\cos \Theta) \sin \Theta \mathrm{d} \Theta \mathrm{d} \varphi=4 \pi
$$

The $\sin \Theta \mathrm{d} \Theta \mathrm{d} \varphi$ weighting takes into account the small solid angles around the "poles" near the forward and backscattering directions, which implies that $E(\chi)$ should be calculated by incorporating the weight measured by the fraction of scattering lights. By simple deduction, the physical weight $\left(\omega^{P}\right)$ is defined by Equation (A5)

$$
\omega_{i}^{P}=\sin \left(\Theta_{i}\right) \Delta \Theta_{i}
$$

Combining $\omega^{M}$ and $\omega^{P}$, the weighing function is expressed by Equation (A6)

$$
\omega_{i}=\sin \left(\Theta_{i}\right) \Delta \Theta_{i} /\left[P_{11}\left(\Theta_{i}\right)\right]^{a}, 0 \leq a \leq 1
$$

The optical $a$ corresponds to the minimum residual, and is determined by an iteration process, where $a$ varies from 0.01 to 1.0 at an increment of 0.11

\section{References}

Baum, B. A., Yang, P., Heymsfield, A. J., Bansemer, A., Cole, B. H., Merrelli, A., Schmitt, C., Wang, C. X., 2014. Ice cloud single-scattering property models with the full phase matrix at wavelengths from 0.2 to 100 um. J. Quant. Spectrosc. Ra., 146: 123-139. http://dx.doi.org/10.1016/j.jqsrt.2014.02.029.

Cox, S. K., 1971. Cirrus Clouds and the Climate. J. Atmos. Sci., 28(8):1513-1514. http://dx.doi.org/10.1175/1520-0469(1971)028<1513:CCATC>2.0.CO;2.

Davis, A. B., Marshak, A., 2001. Multiple scattering in clouds: Insights from three-dimensional diffusion/ $P_{1}$ theory. Nucl. Sci. Eng., 137, 251-280.

http://i3rc.gsfc.nasa.gov/Publications/DavisMarshak_NSE_2001.pdf. 
Davis, A. B., Marshak, A., 2010. Solar radiation transport in the cloudy atmosphere: a 3D perspective on observations and climate impacts. Rep. Prog. Phys., 73, 026801. http://dx.doi.org/10.1088/0034-4885/73/2/026801.

Davis, C. P., Evans, K. F., Buehler, S. A., Wu, D. L., Pumphrey, H. C., 2007. 3-D polarised simulations of space-borne passive mm/sub-mm midlatitude cirrus observations: a case study. Atmos. Chem. Phys., 7 (15): 4149-4158. http://dx.doi.org/10.5194/acp-7-4149-2007.

Davis, A., Marshak, A., Cahalan, R., Wiscombe, W., 1997. The Landsat scale break in stratocumulus as a three-dimensional radiative transfer effect: implications for cloud remote sensing. J. Atmos. Sci., 54 (2): 241-260. http://dx.doi.org/10.1175/1520-0469(1997)054<0241:TLSBIS>2.0.CO;2.

Davis, A., Marshak, A., Wiscombe, W., Cahalan, R., 1996. Scale Invariance of Liquid Water Distributions in Marine Stratocumulus. Part I: Spectral Properties and Stationarity Issues, 53(11): 1538-1558. http://dx.doi.org/10.1175/1520-0469(1996)053<1538:SIOLWD>2.0.CO;2.

Dim, J. R., Takamura, T., Okada, I., Nakajima, T. Y., Takenaka, H., 2007. Influence of inhomogeneous cloud fields on optical properties retrieved from satellite observations. J. Geophys. Res. Atmos., 112(D13), D13202. http://dx.doi.org/10.1029/2006JD007891.

Dunn, M., Johnson, K., Jensen, M., 2011. The Microbase Value-Added Product: A Baseline Retrieval of Cloud Microphysical Properties. U. S. Department of Energy. http://dx.doi.org/10.2172/1015189.

Dupont, J., Haeffelin, M., 2008. Observed instantaneous cirrus radiative effect on surface - level shortwave and longwave irradiances. J. Geophys. Res. Atmos., 113(D21): 6089-6098. http://dx.doi.org/10.1029/2008JD009838.

Evans, K. F., 1998. The spherical harmonics discrete ordinate method for three-dimensional atmospheric radiative transfer. J. Atmos. Sci., 55 (3): 429-446. http://dx.doi.org/10.1175/1520-0469(1998)055<0429:TSHDOM>2.0.CO;2.

Fauchez, T., Cornet, C., Szczap, F., Dubuisson, P., Rosambert, T., 2014. Impact of cirrus clouds heterogeneities on top-of-atmosphere thermal infrared radiation. Atmos. Chem. Phys., 14(11): 5599-5615. http://dx.doi.org/10.5194/acp-14-5599-2014.

Fauchez, T., Dubuisson, P., Cornet, C., Szczap, F., Garnier, A., Pelon, J., Mayer, K., 2015. Impacts of cloud heterogeneities on cirrus optical properties retrieved from space-based thermal infrared radiometry. Atmos. Meas. Tech., 8 (8): 633-647. http://dx.doi.org/10.5194/amt-8-633-2015.

Faure, T., Isaka, H., Guillemet, B., 2001. Neural network analysis of the radiative interaction between neighboring pixels in inhomogeneous clouds. J. Geophys. Res. Atmos., 106(D13): 14465-14484. http://dx.doi.org/10.1029/2000JD900686. 
Garnier, A., Pelon, J., Dubuisson, P., Faivre, M., Chomette, O., Pascal, N., Kratz, D. P., 2012. Retrieval of cloud properties using CALIPSO Imaging Infrared Radiometer. Part I: effective emissivity and optical depth. J. Appl. Meteorol. Clim., 51(7), 1407-1425. http://dx.doi.org/10.1175/JAMC-D-11-0220.1.

Garnier, A., Pelon, J., Dubuisson, P., Yang, P., Faivre, M., Chomette, O., Pascal, N., Lucker, P., Murray, T., 2013. Retrieval of cloud properties using CALIPSO Imaging Infrared Radiometer. part ii: effective diameter and ice water path. J. Appl. Meteorol. Clim., 52(11), 2582-2599. http://dx.doi.org/10.1175/JAMC-D-12-0328.1.

Giuseppe, F. D., 2005. Sensitivity of one-dimensional radiative biases to vertical cloud-structure assumptions: Validation with aircraft data. Q. J. R. Meteorol. Soc., 131(608): 1655-1676. http://dx.doi.org/10.1256/qj.03.129.

Glantz, P., Tesche, M., 2012. Assessment of two aerosol optical thickness retrieval algorithms applied to modis aqua and terra measurements in europe. Atmos. Meas. Tech., 5(7), 1727-1740. http://dx.doi.org/10.5194/amt-5-1727-2012.

Gultepe, I., Starr, D. O'C.,1995. Dynamical structure and turbulence in cirrus clouds: aircraft observations during FIRE. J. Atmos. Sci., 52(23): 4159-4182. http://dx.doi.org/10.1175/1520-0469(1995)052<4159:DSATIC>2.0.CO;2.

Heymsfield, A., 1975. Cirrus uncinus generating cells and the evolution of cirriform clouds. part II: the structure and circulations of the cirrus uncinus generating head. J. Atmos. Sci., 32 (4): 809-819. http://dx.doi.org/10.1175/1520-0469(1975)032<0809:CUGCAT>2.0.CO;2.

Hogan, R. J., Illingworth, A. J., 2003. Parameterizing Ice Cloud Inhomogeneity and the Overlap of Inhomogeneities Using Cloud Radar Data. J. Atmos. Sci., 60(5): 756-767. http://dx.doi.org/10.1175/1520-0469(2003)060<0756:PICIAT>2.0.CO;2.

Hogan, R. J., Kew, S. F., 2005. A 3D stochastic cloud model for investigating the radiative properties of inhomogeneous cirrus clouds. Q. J. R. Meteorol. Soc., 131 (611): 2585-2608. http://dx.doi.org/10.1256/qj.04.144.

Immler, F. J., Dykema, J., Gardiner, T., Whiteman, D. N., Thorne, P. W., Vömel, H., 2010. Reference quality upper-air measurements: guidance for developing gruan data products. Atmos. Meas. Tech., 3(5), 1217-1231. http://dx.doi.org/10.5194/amt-3-1217-2010.

Iwabuchi, H., Hayasaka, T., 2002. Effects of cloud horizontal inhomogeneity on the optical thicknessretrieved from moderate-resolution satellite data. J. Atmos. Sci., 59(14): 2227-2242. http://dx.doi.org/10.1175/1520-0469(2002)059<2227:EOCHIO>2.0.CO;2.

Jensen, E. J., Kinne, S., Toon, O. B., 1994. Tropical cirrus cloud radiative forcing: Sensitivity studies. Geophys. Res. Lett., 21(18): 2023-2026. http://dx.doi.org/10.1029/94GL01358. 
Kablick, I. G. P., Ellingson, R. G., Takara, E. E., Gu, J., 2011. Longwave 3D benchmarks for inhomogeneous clouds and comparisons with approximate methods. J. Clim., 24(8), 2192-2205.

http://dx.doi.org/10.1175/2010JCLI3752.1.

Kalesse, H., 2009. Influence of Ice Crystal Habit and Cirrus Spatial Inhomogeneities on the Retrieval of Cirrus Optical Thickness and Effective Radius, Ph.D. thesis, Johannes Gutenberg University, Mainz, Germany, pp. 65-85.

Kato, S., Marshak, A., 2009. Solar zenith and viewing geometry-dependent errors in satellite retrieved cloud optical thickness: Marine stratocumulus case. J. Geophys. Res. Atmos., 114(D1), D01212. http://dx.doi.org/10.1029/2008JD010579.

Kew, S., 2003. Development of a 3D fractal cirrus model and its use in investigating the impact of cirrus inhomogeneity on radiation, M. S. thesis, Department of Mathematics, University of Reading, pp. 18-24.

Liou, K. N., 1986. Influence of Cirrus Clouds on Weather and Climate Processes: A Global Perspective. Mon. Wea. Rev., 114(6): 1167-1199.

http://dx.doi.org/10.1175/1520-0493(1986)114<1167:IOCCOW>2.0.CO;2.

Mcfarquhar, G. M., Heymsfield, A. J., 1998. The definition and significance of an effective radius for ice clouds, J. Atmos. Sci., 55(11): 2039-2052.

http://dx.doi.org/10.1175/1520-0469(1998)055<2039:TDASOA>2.0.CO;2.

Marshak, A., Davis, A., Wiscombe, W., Cahalan, R., 1998. Radiative effects of sub-mean free path liquid water variability observed in stratiform clouds. J. Geophys. Res. Atmos., 103 (D16): 19557-19567. http://dx.doi.org/10.1029/98JD01728.

Marshak, A., S. Platnick, T. Várnai, G. Wen, Cahalan, R. F., 2006. Impact of three-dimensional radiative effects on satellite retrievals of cloud droplet sizes. J. Geophys. Res., 111, D09207. http://dx.doi.org/10.1029/2005JD006686.

Nazaryan, H., Mccormick, M. P., Menzel, W. P., 2008. Global characterization of cirrus clouds using CALIPSO data. J. Geophys. Res. Atmos., 113 (D16), 280-288. http://dx.doi.org/10.1029/2007JD009481.

Pandey, P., Ridder, K. D., Gillotay, D., Lipzig, N. P. M. V., 2012. Estimating cloud optical thickness and associated surface UV irradiance from SEVIRI by implementing a semi-analytical cloud retrieval algorithm. Atmos. Chem. Phys., 12(1), 7961-7975. http://dx.doi.org/10.5194/acp-12-7961-2012.

Pincus, R., Evans, K. F., 2009. Computational cost and accuracy in calculating three-dimensional radiative transfer: results for new implementations of Monte Carlo and SHDOM. J. Atmos. Sci., 66 (10): 3131-3146. http://dx.doi.org/10.1175/2009JAS3137.1.

Platnick, S., King, M. D., Meyer, K. G., Wind, G., Amarasinghe, N., Marchant, B., Arnold, G. T., Zhang, Z. B., Hubanks, P. A., Ridgway, B. Riedi, J., 2015. MODIS Cloud Optical Properties: User Guide for the Collection 6 Level-2 MOD06/MYD06 Product and Associated Level-3 Datasets, Version 1.0. 
Roebeling, R. A., Feijt, A. J. Stammes, P., 2006. Cloud property retrievals for climate monitoring: implications of differences between SEVIRI on METEOSAT-8 and AVHRR on NOAA-17. J. Geophys. Res. Atmos., 111(D20), D20210. http://dx.doi.org/10.1029/2005JD006990.

Sassen, K., Wang, Z., Liu, D., 2008. Global distribution of cirrus clouds from CloudSat/Cloud-Aerosol lidar and infrared pathfinder satellite observations (CALIPSO) measurements. J. Geophys. Res. Atmos., 113 (D8): 347-348. http://dx.doi.org/10.1029/2008JD009972.

Stubenrauch, C. J., Chédin, A., Rädel, G., Scott, N. A., Serrar, S., 2006. Cloud properties and their seasonal and diurnal variability from TOVS Path-B. J. Clim., 19 (21): 5531-5553. http://dx.doi.org/10.1175/JCLI3929.1.

Szczap, F., Gour, Y., Fauchez, T., Cornet, C., Faure, T., Jourdan, O., Penide, G., Dubuisson, P., 2014. A flexible three-dimensional stratocumulus, cumulus and cirrus cloud generator (3DCLOUD) based on drastically simplified atmospheric equations and Fourier transform framework. Geosci. Model Dev., 7 (1): 1779-1801. http://dx.doi.org/10.5194/gmd-7-1779-2014.

Troyan, D., 2013. Interpolated sounding value-added product. U. S. Department of Energy. DOE/SC-ARM-TR-124. http://www.arm.gov/publications/tech_reports/doe-sc-arm-tr-124.pdf?id=96.

Várnai, T., 1998. Influence of three-dimensional radiative effects on the spatial distribution of shortwave cloud reflection. J. Atmos. Sci., 57(2): 216-229. http://dx.doi.org/10.1175/1520-0469(2000)057<0216:IOTDRE>2.0.CO;2.

Várnai, T., Marshak, A., 2001. Statistical analysis of the uncertainties in cloud optical depth retrievals caused by three-dimensional radiative effects. J. Atmos. Sci., 58 (12): 1540-1548. http://dx.doi.org/10.1175/1520-0469(2001)058<1540:SAOTUl>2.0.CO;2.

Várnai, T., Marshak, A., 2003: A method for analyzing how various parts of clouds influence each other's brightness. J. Geophys. Res., 108 (D22), 4706. http://dx.doi.org/10.1029/2003JD003561.

Wang, C., Platnick, S., Zhang, Z., Meyer, K., Yang, P., 2016. Retrieval of ice cloud properties using an optimal estimation algorithm and MODIS infrared observations: 1 . Forward model, error analysis, and information content. J. Geophys. Res. Atmos., 121(10): 5809-5826. http://dx.doi.org/10.1002/2015JD024526.

Wang, L., Sassen, K., 2008. Wavelet analysis of cirrus multiscale structures from Lidar backscattering: A cirrus uncinus complex case study. J. Appl. Meteor. Climatol., 47(10): 2645-2658. http://dx.doi.org/10.1175/2008JAMC1788.1.

Yamamoto, H., Kamei, A., Nakamura, R., Tsuchida, S., 2011. Long-term cross-calibration of the TERRA ASTER and MODIS over the CEOS calibration sites. Proceedings of SPIE - The International Society for Optical Engineering. 8153, 815318. http://dx.doi.org/10.1117/12.892568. 
Yang P., Bi L., Baum B. A., Liou K. N., Kattawar G. W., Mishchenko M., 2013. Spectrally consistent scattering, absorption, and polarization properties of atmospheric ice crystals at wavelengths from $0.2 \mathrm{~mm}$ to 100 mm. J. Atmos. Sci., 70 (1): 330-347. http://dx.doi.org/10.1175/JAS-D-12-039.1.

Yang, P., Liou, K. N., Bi, L., Liu, C., Yi, B. Q., Baum, B. A., 2015. On the radiative properties of ice clouds: light scattering, remote sensing, and radiation parameterization. Adv. Atmos. Sci., 32 (1): 32-63. http://dx.doi.org/10.1007/s00376-014-0011-z.

Zhang, Z., Ackerman, A. S., Feingold, G., Platnick, S., Pincus, R., Xue, H., 2012. Effects of cloud horizontal inhomogeneity and drizzle on remote sensing of cloud droplet effective radius: case studies based on large-eddy simulations. J. Geophys. Res. Atmos., 117(D19), D19208. http://dx.doi.org/10.1029/2012JD017655.

Zhang, Z., Platnick, S., 2012. An assessment of differences between cloud effective particle radius retrievals for marine water clouds from three MODIS spectral bands. J. Geophys. Res. Atmos., 116(D20), D20215. http://dx.doi.org/10.1029/2011JD016216.

Zhong, W., Hogan, R. J., Haigh, J. D., 2008. Three-dimensional radiative transfer in midlatitude cirrus clouds. Q. J. R. Meteorol. Soc., 134 (630): 199-215. http://dx.doi.org/10.1002/qj.182.

Zinner, T., Mayer, B., 2006. Remote sensing of stratocumulus clouds: uncertainties and biases due to inhomogeneity. J. Geophys. Res. Atmos., 111 (D14209): 257-271. http://dx.doi.org/10.1029/2005JD006955.

Zinner, T., Mayer, B., Schröder, M., 2006. Determination of three-dimensional cloud structures from high-resolution radiance data. J. Geophys. Res. Atmos., 111 (D8), D08204. http://dx.doi.org/10.1029/2005JD006062. 